\title{
HIGHER ORDER RADIATIVE CORRECTIONS AT LEP ENERGIES
}

\author{
F.A. Berends and W.L. van Neerven \\ Instituut-Lorentz, P.0.B. 9506 \\ 2300 RA Leiden, The Netherlands
}

and

G.J.H. Burgers

CERN -- Geneva

\section{A B S T T R A C T}

A complete two-loop $0\left(\alpha^{2}\right)$ initial state radiative correction to the $Z$-resonance shape is presented. The correction is compared with those expressions where only the soft-photon effects are resunmed in all orders of perturbation theory. Our result shows that the soft-photon part constitutes the bulk of the radiative correction near the top of the $Z$ peak. The effect of non-photonic QED processes on the $Z$-resonance is found to be very small. The above results have been obtained by means of a standard Feynman diagram calculation. In addition we have also computed the cross-sections by using the renormalization group method, where besides the leading logs $\ln \left(\mathrm{s} / \mathrm{m}^{2}\right)$, also the next-to-leading ones have been taken into account. 


\section{1. - INTRODUCTION}

Electron-positron colliding beam experiments which will be carried out in the near future at SLC and LEP will provide us with a wealth of information about the standard model of the electroweak interactions. A vast amount of literature ${ }^{1)}$ exists about the subjects one wants to investigate. We just mention topics such as the search for new particles like the Higgs boson, the top quark and maybe some supersymmetric partners of the particles in the standard model. Furthermore one wants to make precise determinations of the electroweak parameters among which the most interesting are the mass and width of the $\mathrm{Z}$ boson. For the study of new physics effects, it is of the utmost importance to measure these quantities with a very high degree of accuracy. As has been extensively discussed in Refs. 2) and 3), the determination of the mass and width of the $z$ boson will be greatly affected by radiative corrections. In particular the pure QED part of the standard model leads to a distortion and a shift of the resonance peak. Up to now complete one-loop radiative corrections of the process $\mathrm{e}^{+} \mathrm{e}^{-} \rightarrow \mu^{+} \mu^{-}$in which the $\mathrm{Z}$ is produced have been carried out ${ }^{4)}$. The calculations reveal that the bulk of the corrections can be attributed to photonic contributions from the initial electron-positron state. Exponentiation of the lowest-order soft photon contribution shows ${ }^{2), 3)}$ that higher-order radiative corrections, in particular for initial state radiation processes, will become noticeable. Because of the possibility that one will measure the shape of the Z-resonance with very high precision $\left(\delta m_{z} \approx \delta \Gamma_{\text {tot }} \approx \pm 10 \mathrm{MeV}\right)^{3)}$ one has to investigate the validity of the soft photon approximation and resummation. Therefore one has to include hard photon radiative contributions and other production mechanisms of the $z$ boson, e.g., processes where additional lepton pairs are produced. Besides their importance for an accurate measurement of the electroweak parameters, the radiative corrections are also relevant for other areas in collider physics. In particular they show up in the determination of the quantity $R=\sigma_{e^{+} e^{-} \rightarrow x} / \sigma_{e^{+} e^{-} \rightarrow \mu^{+} \mu^{-}}^{(0)}$ where $X$ represents all hadronic final states.

In this paper we shall limit ourselves to the pure QED initial state part of the radiative corrections to z-production. This implies that we shall also omit all electroweak corrections to the $Z$ propagator which have to be taken into account for the determination of its mass and width. However, the most important effects can easily be incorporated in our calculations. In this work we present an analytic calculation of the cross-section do/ds' up to order $\alpha^{4}$ where $s^{\prime}$ is the invariant di-muon pair mass. Of course, it is very well understood that for any experimental setup one has to introduce cuts on energies and angles which 
can most easily be achieved by an event generator ${ }^{5)-7)}$. Nevertheless an analytic formula for the quantity $\mathrm{d} \sigma / \mathrm{ds}$ ' provides us with some useful information about the order of magnitude of the various $z$-production mechanisms. It is also useful for an event generator for neutrino counting ${ }^{8)} e^{+} e^{-} \rightarrow \nu \bar{\nu} \gamma(\gamma)$ which we plan to construct in the near future. Finally an analytic expression allows us to make a good prediction for the shift and distortion of the $Z$-peak yielding an accurate determination of the mass and width of the $Z$ boson.

Our limitation to the pure QED part has the advantage that we can avoid the awkward choice of the various renormalization schemes which play such an important rôle in the standard model. This is because the coupling constant which describes the interaction between the $\mathrm{Z}$ and the incoming and outgoing leptons will not be renormalized by pure QED corrections. Therefore we only have to deal with the renormalization of the fine structure constant which will be carried out in the standard way as has been described in many textbooks. Another advantage is that the radiative corrections in QED only contain two mass scales, namely $m_{e}$ and $M_{Z}$, which give rise to logarithmic enhanced terms in the cross-section of the type $\ln { }^{i} \mathrm{M}_{\mathrm{Z}}^{2} / \mathrm{m}_{\mathrm{e}}^{2}$. The latter allows us to apply renormalization group methods which lead to reasonable estimates for $d \sigma / d s^{\prime}$. This has been done in the leading $10 g$ approximation in Refs. 9)-12) and will be extended to the next-to-leading logarithms in Chapter 4.

In studying $\mathrm{Z}$-production, one distinguishes two types of QED corrections [for the nomenclature, see Ref. 13)].

A) Photonic corrections. Here one adds extra photon lines to the Born graph in Fig. 1 without including addition lepton pairs.

B) Non-photonic corrections. They include contributions from additional lepton pairs in the QED case considered.

In case $A$ ), we shall limit ourselves to initial-state radiation including the accompanying virtual corrections since only the latter process gives rise to large logarithmic terms appearing in the total cross-section. However, one has to bear in mind that contributions from final-state radiation can be of the same order as the non-logarithmic terms. If it turns out that in the latter case the non-logarithmic terms give a noticeable contribution then also final-state 
radiation has to be considered. This in particular holds if one wants to calculate the forward-backward asymmetry where, e.g., the box graphs play an important rôle.

The paper is organized as follows. In section 2 we present the results of a ful1 two-loop initial-state order $\alpha^{2}$ calculation to the Born cross-section of the process $e^{+} e^{-} \rightarrow \mu^{+} \mu^{-}$(Fig. 1) due to contributions of the type A) and B). In Section 3 we discuss the contribution of soft-photon and fermion-pair radiation to $Z$ production. In Section 4 , it is shown that all the logarithmic enhanced terms in the cross-section can be obtained by using renormalization group methods. In section 5, the effect of the higher-order radiative corrections on the shape of the $z$-peak and the determination of the mass and width will be discussed. The technical details of our calculations will be presented in Appendices $A$ and $B$.

\section{2. - THE FULL $O\left(\alpha^{2}\right)$ CALCULATION}

In this section we will present the results of the second-order radiative correction in $\alpha$ to the Born cross-section of the process in Fig. 1

$$
e^{+}\left(p_{+}\right)+e^{-}\left(p_{-}\right) \rightarrow V(q) \rightarrow \mu^{+}\left(q_{+}\right)+\mu^{+}(q)(2.1)
$$

where $\mathrm{V}$ denotes the neutral electroweak vector bosons of the standard model which are represented by $Z$ and the virtual photon $\gamma^{*}$. The photonic correction $A$ ) due to initial-state radiation is given by the process

$$
\begin{aligned}
e^{+}\left(p_{+}\right)+e^{-}\left(p_{-}\right) \rightarrow & V(q)+\gamma\left(k_{1}\right)+\gamma\left(k_{2}\right) \\
& \longrightarrow \mu^{+}\left(q_{+}\right)+p^{-}\left(q_{-}\right)
\end{aligned}
$$

Since the cross-section of the above process is of the order $\alpha^{4}$ we have also to consider another process which belongs to category B) and contributes in the same order of perturbation theory. It is the reaction where an additional lepton pair is produced

$$
\begin{aligned}
e^{+}\left(p_{+}\right)+e^{-}\left(p_{-}\right) \rightarrow & V(q)+f^{+}\left(k_{1}\right)+f^{-}\left(k_{2}\right) \\
& L^{+}\left(q_{+}\right)+\mu-\left(q_{-}\right)
\end{aligned}
$$

As has been mentioned in the Introduction, we shall limit ourselves in Eq. (2.2) to initial-state two-photon radiation which includes all the corresponding 
virtual corrections. Therefore both reactions in Eqs. (2.2) and (2.3) can be described by the Drell-Yan mechanism which has been developed in the context of perturbative QCD. The similarity between the Drell-Yan process for parton-parton interactions in $Q C D$ and the radiative corrections in $Q E D$ has been extensively treated in Refs. 10)-12) and we will elaborate more upon this subject in Section 4 .

In this paper we shall give an exact analytic expression for the crosssection $d \sigma / d s^{\prime}$ in the limit $s^{\prime}, s \gg \mathrm{m}^{2}$ where $m$ denotes the electron mass. $s=\left(p_{+}+p_{-}\right)^{2}$ is the centre-of-mass energy squared and the invariant mass of the muon paix will be denoted by $s^{\prime}=\left(q_{+}+q_{-}\right)^{2}$. According to the Drell-Yan mechanism the unpolarized cross-section $d \sigma / d s^{\prime}$ will be given by the following expression

$$
\begin{aligned}
& \frac{d \sigma}{d s^{\prime}}=\sigma^{(0)}\left(s^{\prime}\right) \frac{1}{4 s} \sum_{n=0}^{\infty} \int d^{4} q \delta\left(q^{2}-s^{\prime}\right) \frac{1}{(2 n)^{3 n}} \prod_{i=1}^{n} d^{4} k_{i} \delta\left(k_{i}^{2}-m_{i}^{2}\right) \\
& \vartheta\left(k_{i}^{0}\right) \delta^{4}\left(p_{+}+p_{-}-q-\sum_{j=1}^{n} k_{j}\right)\left|T^{(n)}\right|^{2}
\end{aligned}
$$

where $k_{i}, m_{i}$ represent the momenta and masses of the particles in the final state. $\sigma^{(0)}\left(s^{\prime}\right)$ stands for the lowest-order total cross-section of the process in Eq. (2.1) which is equal to

$$
\sigma^{(0)}\left(s^{\prime}\right)=\frac{4 \pi \alpha^{2}}{3 s^{\prime}} P\left(s^{\prime}, m_{p}^{2}\right)\left[1+c_{v}^{2} \frac{2\left(s^{\prime}-M_{z}^{2}\right) s^{\prime}}{\left|Z\left(s^{\prime}\right)\right|^{2}}+\left(c_{v}^{2}+c_{A}^{2}\right)^{2} \frac{s^{\prime 2}}{\left|Z\left(s^{\prime}\right)\right|^{2}}\right]_{(2.5)}
$$

with

$$
\left.P\left(s^{\prime}, m_{r}^{2}\right)=\left(1+\frac{2 m_{r}^{2}}{s^{\prime}}\right) \sqrt{1-\frac{4 m_{c}^{2}}{s^{\prime}}}, \quad Z s^{\prime}\right)=s^{\prime}-M_{z}^{2}+i M_{z} \Gamma_{z}
$$

where $M_{Z}, \Gamma_{Z}$ denote the mass and total width of the $Z$-boson. The $Z-\ell^{+}-\ell^{-}$coupling constants are given by

$$
C_{A}=\frac{-1}{2 \sin 2 \theta_{w}} \quad C_{V}=C_{A}\left(1-4 \sin ^{2} \theta\right)
$$

Furthermore, expression (2.4) is written in such a way that $T^{(n)}$ is the amplitude of the process where $n$ is equal to the number of particles (not the muon pair) in the final state. Notice that Eq. (2.4) is correct provided we neglect terms of ordex $\mathrm{m}^{2} / \mathrm{s}$ in the cross-section. As has been mentioned in the Introduction, the coupling constant which describes the interaction of the $z$ boson with the initial state electron-positron pair will not be renormalized by the radiative 
corrections of type A) and B) to process (2.1). This is due to the conservation of the neutral weak current in the 1 imit $m \rightarrow 0$. The fact that in the 1 imit $m \rightarrow 0$ $T^{(n)}[\mathrm{Eq} .(2.4)]$ for $z$ production is the same as for the intermediate virtual photon processes follows from the universality of collinear divergences (mass singularities) in quantum field theory. This property has been verified in our calculations which are given below.

$T^{(n)}$ will be given by the standard Feymman rules which include a statistical factor in the case of identical particles in the final state. In Appendix A we have given an outline of our techniques for the process $e^{+} e^{-} \rightarrow \mu^{+} \mu^{-}+\gamma+\gamma$. The cross-sections for the other processes can be obtained in an analogous way. For more details, in particular for the process in Eq. (2.3), see Ref. 14). In our subsequent discussion, we call the photonic contribution A) process $I$, and the non-photonic ones B) will be subdivided into processes II, III and IV corresponding to their different production mechanisms.

Our results can be summarized as follows.

In lowest order $\left(\alpha^{2}\right)$ the cross-section of the process in Fig. 1

$$
e^{+}\left(p_{+}\right)+e^{-}\left(p_{-}\right) \rightarrow \mu^{+}\left(q_{+}\right)+\mu^{-}\left(q_{-}\right)
$$

is equal to

$$
\frac{d \sigma_{e^{+} e^{-}}^{(0), I}}{d s^{\prime}}=\delta\left(s-s^{\prime}\right) \sigma^{(0)}\left(s^{\prime}\right)
$$

The first-order radiative correction to the process in Eq. (2.8) is given by the reaction in Fig. $2^{5), 6)}$

$$
e^{+}\left(p_{+}\right)+e^{-}\left(p_{-}\right) \rightarrow \mu^{+}\left(q_{+}\right)+\mu^{-}\left(q_{-}\right)+\gamma\left(k_{1}\right)
$$

where the photon is only radiated off the leptons in the initial state. The cross-section consists of three parts:

$$
\frac{d \sigma_{e+e^{-}}^{(1) I}}{d s^{\prime}}=\frac{1}{s} \sigma^{(0)}\left(s^{\prime}\right)\left[\delta\left(1-s^{\prime} / s\right)\left(\delta_{1}^{s_{1}}(\lambda, \varepsilon)+\delta_{1}^{V_{1}}(\lambda)\right)+\vartheta\left(1-s^{\prime} / s-\varepsilon\right) \delta_{1}^{H_{1}}\left(\frac{s^{\prime}}{s}\right)\right]
$$

The soft photon part equals (Fig. 2) 
$-6-$

$$
\delta_{1}^{S_{1}}(\lambda, \varepsilon)=\frac{\alpha}{\pi}\left[\frac{1}{2} L^{2}-\ln \frac{\lambda^{2}}{m^{2}} L+2 \ln \varepsilon L+\ln \frac{\lambda^{2}}{m^{2}}-2 \ln \varepsilon-23(2)\right]_{(2.12)}
$$

with

$$
L=\ln \frac{s}{m^{2}} \quad, \quad \varepsilon=\frac{2 \Delta}{\sqrt{s}}<<1
$$

$\zeta(n)$ denotes the Riemann zeta function, $\zeta(2)=\pi^{2} / 6, \zeta(3) \approx 1.202 . \Delta$ stands for the energy cutoff on the soft-photon bremsstrahlung integrals which separates the cross-section into a soft $\left(k_{i_{0}}\langle\Delta)\right.$ and a hard $\left.k_{i o}>\Delta\right)$ photon part. $\lambda(\lambda<<m)$ is a photon mass which has to be introduced in order to regulate the infrared divergences which emerge from the phase space integrals in Eq. (2.4). Notice that $\Delta$ in Eq. (2.13) and in the subsequent equations can be taken arbitrary with respect to the electron mass. Therefore it can be used as the experimental energy resolution in order to distinguish between soft and hard photons. More about the meaning and use of the parameter $\Delta$ can be found in Appendix $B$.

The same type of infrared divergences which appear in $\delta_{1}^{S_{1}}$ show up also in the virtual photon corrections to the lowest order cross-section in Eq. (2.9) (Fig. 3). The virtual part $\delta_{1}^{V}$ equals

$$
\delta_{1}^{V_{1}}(\lambda)=2 \operatorname{Re} F^{(1)}(s)
$$

where $F^{(1)}(s)$ is the lowest-order electron form factor (Fig. 3). For $s>m^{2}$ it is given by

$$
\begin{aligned}
F^{(1)}(s)= & \frac{\alpha}{\pi}\left[-\frac{1}{4} L^{2}+\frac{1}{2} \ln \frac{\lambda^{2}}{m^{2}} L+\frac{3}{4} L-\frac{1}{2} \ln \frac{\lambda^{2}}{m^{2}}-1+23(2)\right. \\
& \left.+i \pi\left\{\frac{1}{2} L-\frac{1}{2} \ln \frac{\lambda^{2}}{m^{2}}-\frac{3}{4}\right\}\right]
\end{aligned}
$$

The hard photon part $\delta_{1}^{\mathrm{H} l}$ is equal to (Fig. 2)

$$
\delta_{1}^{H_{1}}\left(s^{\prime} / s\right)=\frac{\alpha}{\pi}\left[\frac{1+z^{2}}{1-z}(L-1)\right]
$$

with

$$
z=5 / s \text { and } 1-\varepsilon>z>\frac{4 m_{\nu}{ }^{2}}{s}
$$


Notice that the infra-red singular terms $\ln \left(\lambda^{2} / \mathrm{m}^{2}\right)$ will be cancelled by adding the soft $\delta_{1}^{S_{1}}$ [Eq. (2.12)] and virtual $\delta_{1}^{V}$ [Eq. (2.14)] parts, whereas the $\ln \varepsilon$ terms disappear if expression (2.11) is integrated over $s^{\prime}$.

The presentation of the second-order radiative correction proceeds in the same way as above. The reaction under consideration is given by (Fig. 4)

$$
e^{+}\left(p_{+}\right)+e^{-}\left(p_{-}\right) \rightarrow \mu^{+}\left(q^{+}\right)+\mu^{-}\left(q_{-}\right)+\gamma\left(k_{1}\right)+\gamma\left(k_{2}\right)
$$

In order to give a clear presentation of the separate contributions to the process in Eq. (2.18) we decompose the cross-section into six parts, i.e.,

$$
\begin{aligned}
\frac{d \sigma_{e^{+} e^{-}}^{(2) I}}{d s^{\prime}} & =\frac{1}{s} \sigma^{(-)}\left(s^{\prime}\right)\left[\delta\left(1-s^{\prime} / s\right)\left\{\delta_{2}^{S_{2}}(\varepsilon, \lambda)+\delta_{2}^{V_{2}}(\lambda)+\delta_{2}^{s_{1}^{\prime} V_{1}}(\varepsilon, \lambda)\right\}+\right. \\
& \left.\vartheta\left(1-s^{\prime} / s-\varepsilon\right)\left\{\delta_{2}^{s_{1} H_{1}}\left(\varepsilon, \lambda, s^{\prime} / s\right)+\delta_{2} V_{1} H_{1}\left(\lambda, s^{\prime} / s\right)+\delta_{2}^{H} H_{2}\left(\varepsilon, s^{\prime} / s\right)\right\}\right]
\end{aligned}
$$

For convenience, we have taken the energy cut-off $\Delta$ equal for both photons so that $\varepsilon_{1}=\varepsilon_{2} \equiv \varepsilon$. The contributions $\delta_{2}$ have the following meaning:

$\delta_{2}^{S_{2}}$ both photons are soft, i.e., $\mathrm{k}_{10}+\mathrm{k}_{20}<\Delta$,

$\delta_{2}$ both photons $\mathrm{k}_{1}$ and $\mathrm{k}_{2}$ are virtual,

$\delta_{2} S_{1} V_{1}$ one photon is soft $k_{10}<\Delta$ and the other photon $k_{2}$ is virtual and vice versa,

$\delta_{2}^{S_{1} H_{1}}$ one photon is soft $k_{10}<\Delta$ and the other photon $k_{2}$ is hard and vice versa,

$\delta_{2}^{V} l_{l}$ one photon $k_{1}$ is virtual and the other one is hard $k_{20}>\Delta$ and vice versa,

$\delta_{2}^{\mathrm{H} 2}$ both photons are hard, i.e., $\mathrm{k}_{10}>\Delta$ and $\mathrm{k}_{20}>\Delta$.

The expression for $\delta_{2}^{S_{2}}$ is given by

$$
\delta_{2}^{s_{2}}(\varepsilon, \lambda)=\frac{1}{2}\left(\delta_{1} s_{i}(\lambda, \varepsilon)\right)^{2}+c_{2}
$$

with $\delta_{1}^{S_{1}}(\varepsilon, \lambda)$ as defined in Eq. (2.12). C 2 is a correction factor whose meaning will be explained in Appendix B. It equals

$$
c_{2}=\left(\frac{\alpha}{\pi}\right)^{2}\left[-2 \zeta(2)(L-1)^{2}\right]
$$


It is a remarkable property of this process that the total soft-collinear part is given by expression (2.20). It indicates that the soft-collinear divergences in higher-order perturbation theory can be obtained by exponentiating the lowest-order correction term $\delta_{1}^{S l}$ [Eq. (2.12)] apart from a correction factor $c_{n}$ which is a generalization of $c_{2}$ (for its explanation, see Refs. 10), 15) and Appendix B]. This property is characteristic for the on-shell mass assignment used in the calculation of the Drell-Yan type of process in Eq. (2.2) and the fact that we work in the centre-of-mass frame of the incoming electron-positron pair. Notice that in general only the higher order infra-red divergences represented by the terms $\ln \left(\lambda^{2} / \mathrm{m}^{2}\right)$ can be obtained via exponentiation of $\left.\delta_{1}^{S_{1}} 16\right)$. The virtual part is given by the Feynman diagrams in Fig. 5. $\delta_{2}^{\mathrm{V}}(\lambda)$ is now given by

$$
\delta_{2}^{V_{2}}(\lambda)=\left|F^{(1)}(s)\right|^{2}+2 \operatorname{Re} F^{(2)}(s)
$$

where $F^{(1)}(s)$ is defined by Eq. (2.15) and for $\left.s \gg m^{2} \operatorname{Re} F^{(2)}(s) 17\right), 18$ ) equals

$$
\begin{aligned}
& \text { Re } F^{(2)}(5)=\left(\frac{\alpha}{\pi}\right)^{2}\left[\frac{1}{32} L^{4}-\frac{3}{16} L^{3}+\left(\frac{7}{32}-\frac{5}{4} 3(2)\right) L^{2}+\right. \\
& \left(-\frac{21}{32}+33(2)+\frac{3}{2} 3(3)\right) L+\frac{2}{5} 3(2)^{2}-\frac{9}{4} \zeta(3)-33(2) \ln 2-\frac{1}{2} 3(2)+\frac{4 \cdot 5}{2 \cdot 6} \\
& \left.+\ln ^{2} \frac{\lambda^{2}}{m^{2}}\left(\frac{1}{8} L^{2}-\frac{1}{4} L+\frac{1}{8}-\frac{3}{4} 3(2)\right)+\ln \frac{\lambda^{2}}{m^{2}}\left(-\frac{1}{8} L^{3}+\frac{1}{2} L^{2}+\left(-\frac{7}{8}+\frac{5}{2} 3(2)\right) L+\frac{1}{2}-\frac{13}{4} 3(2)\right)\right]
\end{aligned}
$$

The next part is obtained from Fig. 6. One can show that for $\Delta \ll E \equiv \frac{1}{2} \sqrt{ }$, $\delta_{2}^{S}{ }_{1} V_{1}(\varepsilon, \lambda)$ factorizes irrespective of whether $\Delta$ is smaller or larger than $\mathrm{m}$. It is equal to [see Eqs. (2.12) and (2.14)]

$$
\delta_{2} S_{1} V_{2}(\varepsilon, \lambda)=\delta_{1} S_{1}(\lambda, \varepsilon) \delta_{1} V_{1}(\lambda)
$$

The same holds for $\delta_{2}^{S_{1} H_{1}}$ [see Eqs. (2.12), (2.16) and Fig. 4]

$$
\delta_{2} S_{1} H_{1}(\varepsilon, \lambda, s \% s)=\delta_{1} S_{1}(\lambda, \varepsilon) \delta_{1}^{H_{1}}\left(s^{\prime} / s\right)
$$

The expression where one photon is virtual and the other one is hard has been calculated in Ref. 19) (Fig. 6). It is equal to:

$$
\begin{aligned}
& \delta_{2}^{V_{1} H_{1}}\left(\lambda, s^{\prime} / s\right)=\delta_{1}^{V_{1}}(\lambda) \delta_{1}^{+1_{1}}\left(s^{1} / s\right)+\left(\frac{\alpha}{\pi}\right)^{2}\left[-\frac{1}{2} \frac{1+z^{2}}{1-z} \ln z L^{2}+\right. \\
& \left\{\frac{1+z^{2}}{1-z}\left(\lim _{2}(1-z)+\ln z \ln (1-z)-\frac{1}{2} \ln ^{2} z+\frac{5}{2} \ln z\right)-(1-z) \ln z+\frac{1}{2} z\right\} L+
\end{aligned}
$$




$$
\begin{aligned}
& \frac{1+z^{2}}{1-z}\left(S_{1,2}(1-z)+\ln z \ln 2(1-z)-\frac{1}{6} \ln ^{3} z+\frac{1}{2} \ln ^{2} z \ln (1-z)-\frac{3}{2} \ln z \ln (1-z)-\frac{3}{2} \lim (1-z)\right. \\
- & \left.4 \ln z+\frac{1}{2} \ln ^{2} z\right)+(1+z)\left(2 \operatorname{li} 3(1-z)-S_{, 2}(1-z)-\ln (1-z) \ln i_{2}(1-z)\right. \\
& \left.+\frac{1}{4} \ln ^{2} z\right)+\frac{1}{2}(1-7 z)(\operatorname{lin}(1-z)+\ln z \ln (1-z))-(1-5 z)\left(\frac{1}{4} \ln ^{2}(1-z)+3(2)\right. \\
& \left.+\frac{1}{2}(3-2 z) \ln (1-z)+\frac{1}{2}(7-5 z) \ln z-1\right]
\end{aligned}
$$

where $\delta_{1}^{\mathrm{V}} \mathrm{l}(\lambda)$ and $\delta_{1}^{\mathrm{H}_{1}}$ are defined by Eqs. (2.14) and (2.16) respectively. Further the functions $\operatorname{Li}_{n}(x)$ and $s_{n, p}(x)$ represent the polylogarithms which $c$ an be found in Refs. 17) and 20).

Equations (2.25) and (2.26) have an interesting property if we take the limit $s^{\prime} \rightarrow 0(2.10)$ and replace $\ln z \rightarrow-$ L. Here we obtain

$$
\delta_{2}^{S_{1} H_{1}}+\delta_{2}^{V_{1} H_{1}} \underset{z \rightarrow 0}{\longrightarrow}\left(\frac{\alpha}{n}\right)^{2}\left[2 \ln \varepsilon(L-1)^{2}+\frac{1}{6} L^{3}+\frac{3}{4} L^{2}+(23(2)-3) L+\theta(1)\right]
$$

which is just the order $\alpha$ virtual correction to the process $e^{+} e^{-} \rightarrow \gamma \gamma$ which has been calculated in Refs. 21). This property can be attributed to the fact that we

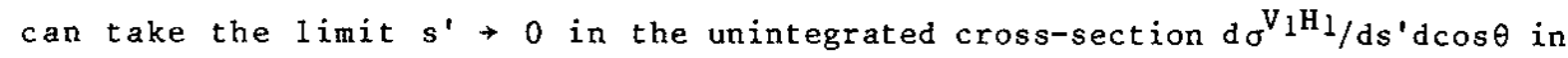
Eq. (4) of Ref. 19). The reason that this limit can be taken is due to another property which can be described as follows. Suppose a gauge boson like the $Z$ or $\gamma^{*}$ is an external leg of a virtual graph and is coupled to a spin- $\frac{1}{2}$ or spin-zero internal particle $m_{i}$. Then because of the vector coupling of the gauge boson to that particle, the collinear divergence which arises in the limits $m_{i} \rightarrow 0, s^{\prime} \rightarrow 0$ $\left(s^{\prime}=\right.$ mass of the gauge boson) appears in the structure function multiplying the momentum of the gauge boson (here $q_{\mu}$ ). If we now compute $\left|T^{(n)}\right|^{2}$ this momentum will be contracted with a gauge-invariant set of graphs, so that this structure function does not show up in the final result. The vanishing of this collinear divergence implies that the order of the limits $m_{i} \rightarrow 0$ and $s^{\prime} \rightarrow 0$ becomes irrelevant. The same property shows up if $m_{i}$ is not an internal particle but represents the cutted line in a cut-graph [for a definition of a cut-graph see Ref. 22) ]. The additional mass singular term which emerges from the limit $z \rightarrow 0$

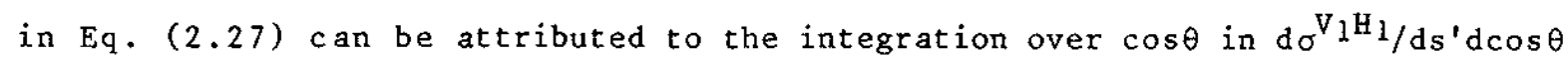
and represents an initial state collinear divergence. 
The contribution to the cross-section where both photons are hard has been calculated in Appendix A [see also Ref. 23)]. Its result is (Fig. 4)

$$
\begin{aligned}
& \delta_{2}^{H_{2}}\left(\varepsilon, S^{\prime} / s\right)=\left(\frac{\alpha}{n}\right)^{2}\left[\left\{\frac{1+z^{2}}{1-z}\left(2 \ln \left(\frac{1-z}{\varepsilon}\right)-\frac{1}{2} \ln z\right)-(1-z)+\frac{1}{2}(1+z) \ln z\right\} L^{2}\right. \\
& +\left\{\frac{1+z^{2}}{1-z}\left(-4 \ln \left(\frac{1-z}{\varepsilon}\right)+\ln z\right)-z \ln z+\frac{z}{2}(1-z)+\frac{1}{4}(1+z) \ln ^{2} z\right\} L+ \\
& \quad \frac{1+z^{2}}{1-z}\left(-S_{1,2}(1-z)-\frac{1}{2} \ln z \operatorname{lin}(1-z)-\frac{3}{2} \ln ^{2} z+\left(3(2)+\frac{z}{6}\right) \ln z+2 \ln \left(\frac{1-z}{\varepsilon}\right)\right) \\
& \left.-(1+z)\left(\frac{1}{2} \lim (1-z)+S_{1,2}(1-z)\right)-\left(\frac{1}{2}+\frac{2}{3} z\right) \operatorname{li} 2(1-z)-\frac{z}{3} z\right\}(z)-\frac{1}{6}(10-25 z) \ln z \\
& \left.+\frac{2}{(1-z)^{2}} \ln ^{2} z-\left(\frac{z}{3} z+\frac{1}{4}\right) \ln ^{2} z+\frac{1}{2}(1-z)-\frac{2}{3} \frac{z}{1-z}\left(1+\frac{2}{1-z} \ln z+\frac{1}{(1-z)^{2}} \ln ^{2} z\right)\right]
\end{aligned}
$$

The terms $\ln ((1-z) / \varepsilon)$ originate from the infra-red behaviour of the threeparticle phase space integrals given in Eqs. (A.31)-(A.38). They arise because one of the photon vector-boson invariants $s_{3}, s_{4}$ [Eq. (A.4)] has to be taken larger than $s^{\prime}$ [see Eqs. (A.9), (A.10) and the Dalitz plot in Fig. 7]. Apart from these terms neither $\delta_{l}^{\mathrm{H}}{ }_{1}$ [Eq. (2.16)] nor $\delta_{2}^{\mathrm{H}} 2$ [Eq. (2.28)] contain functions of the type $\ln (1-z)$ which is a characteristic feature of the our calculation where the initial-state fermions in Eqs. (2.10) and (2.18) are taken on shell. Finally, we want to comment on the limit $s^{\prime} \rightarrow 0(z \rightarrow 0)$ of Eq. (2.28). In this limit we get

$\delta_{2}^{H_{2}} \underset{\substack{z \rightarrow 0 \\ h \rightarrow-L}}{\longrightarrow}\left(\frac{\alpha}{\pi}\right)^{2}\left[-2 \ln \varepsilon(L-1)^{2}+\frac{1}{4} L^{3}-\frac{7}{4} L^{2}+\left(-\frac{1}{2} 3(2)+4\right) L+\theta(1)\right]$

Apart from the coefficient of the term ln $\varepsilon$, the expression in Eq. (2.29) disagrees with the result obtained for the total cross-section of the process $e^{+} e^{-} \rightarrow \gamma \gamma \gamma$ given in Ref. 21). This is because the gauge boson is not coupled to a particle which corresponds to an internal line of a virtual graph or a cut line. Therefore, the property as described below [Eq. (2.27)] will not hold.

Summarizing the results obtained above, we are now able to give the order $\alpha^{2}$ radiative correction to process $I$ which is due to initial state photon radiation 23$), *)$. The division in $\delta^{\mathrm{V}+\mathrm{S}}$ and $\widetilde{\delta}^{\mathrm{H}}$ parts will be used in section 3 .

*) Notice that there is a misprint in Eq. (14) of Ref. 23), $-\ln { }^{2} z / v$ should be $+\ln ^{2} \mathrm{z} / \mathrm{v}$. In Eq. (15), the square brackets should be omitted. 
- 11 -

$$
\begin{aligned}
& \frac{d \sigma_{e^{+} e^{-}}^{(2), I}}{d s^{\prime}}=\frac{1}{s} \sigma^{(0)}\left(s^{\prime}\right)[\delta(1-z)+ \\
& \left(\frac{\alpha}{\pi}\right)\left[\delta(1-z)\left[2(L-1) \ln \varepsilon+\left\{\frac{3}{2} L+2 \zeta(2)-2\right\}\right]+\vartheta(1-z-\varepsilon)\left[2(L-1) \frac{1}{1-z}-(1+z)(L-1)\right]\right] \\
& +\left(\frac{\alpha}{\pi}\right)^{2}\left[\delta ( 1 - z ) \left[2(L-1)^{2} \ln ^{2} \varepsilon+2(L-1) \ln \varepsilon\left\{\frac{3}{2} L+23(2)-2\right\}+\left\{(9 / 8-23(2)) L^{2}+\right.\right.\right. \\
& \left.\left.\left(\frac{45}{16}+\frac{11}{2} 3(2)+33(3)\right) L-6 / 53(2)^{2}-9 / 23(3)-63(2) \ln 2+3 / 83(2)+\frac{513}{108}\right\}\right]+ \\
& \vartheta(1-z-\varepsilon)\left[4(L-1)^{2} \frac{\ln (1-z)}{1-z}+2(L-1) \frac{1}{1-z}\left\{\frac{3}{2} L+23(2)-2\right\}+\left\{\left(-\frac{1+z^{2}}{1-z} \ln z+\right.\right.\right. \\
& \left.(1+z)\left(-2 \ln (1-z)+\frac{1}{2} \ln z\right)-5 / 2-1 / 2 z\right) L^{2}+\left(\frac { 1 + z ^ { 2 } } { 1 - z } \left(\operatorname{li} 2(1-z)+\ln z \ln (1-z)+\frac{z}{2} \ln z\right.\right. \\
& \left.\left.-\frac{1}{2} \ln ^{2} z\right)+(1+z)\left(\frac{1}{4} \ln ^{2} z+4 \ln (1-z)-23(2)\right)-\ln z+z+\frac{1}{2} z\right) L+\frac{1+z^{2}}{1-z}\left(-\frac{1}{6} \ln ^{3} z+\right. \\
& \left.\frac{1}{2} \ln z \operatorname{lin}(1-z)+\frac{1}{2} \ln ^{2} z \ln (1-z)-\frac{3}{2} \operatorname{li} 2(1-z)-\frac{3}{2} \ln z \ln (1-z)+3(z) \ln z-\frac{1}{6} \ln z-\ln ^{2} z\right)+ \\
& (1+z)\left(\frac{3}{2} \operatorname{lig}_{3}(1-z)-2 S_{1,2}(1-z)-\ln (1-z) \operatorname{hi}(1-z)-5 / 2\right)-\frac{1}{4}(1-5 z) \ln ^{2}(1-z)+ \\
& +\frac{1}{2}(1-7 z) \ln z \ln (1-z)-\frac{25}{6} z \lim _{2}(1-z)+\left(1+\frac{19}{3} z\right) 3(2)+\left(-\frac{1}{2}-3 z\right) \ln (1-z)+\frac{1}{6}(11+10 z) \ln z \\
& \left.\left.\left.\frac{2}{(1-z)^{2}} \ln ^{2} z-\frac{25}{12} z \ln ^{2} z-\frac{2}{3} \frac{z}{1-z}\left(1+\frac{2}{1-z} \ln z+\frac{1}{(1-z)^{2}} \ln ^{2} z\right)\right\}\right]\right] \\
& =\frac{1}{s} \sigma^{(s)}\left(s^{\prime}\right)[\delta(1-z)+
\end{aligned}
$$

$$
\begin{aligned}
\left(\frac{\alpha}{n}\right) & {\left[\delta(1-z)\left[2(L-1) \ln \varepsilon+\delta_{1}^{V+S}\right]+\vartheta(1-z-\varepsilon)\left[2(L-1) \frac{1}{1-z}+\tilde{\delta}_{1}^{H}\right]\right.} \\
+\left(\frac{\alpha}{n}\right)^{2} & {\left[\delta(1-z)\left[2(L-1)^{2} \ln ^{2} \varepsilon+2(L-1) \ln \varepsilon \delta_{1}^{V+S}+\delta_{2} V+S\right]+\right.} \\
& \left.\vartheta(1-z-\varepsilon)\left[4(L-1)^{2} \frac{\ln (1-z)}{1-z}+2(L-1) \frac{1}{1-z} \delta_{1} V+S+\tilde{\delta}_{2}^{H+1}\right]\right]
\end{aligned}
$$

$(2.30)$

In the last part of this section, we shall discuss the processes where an additional lepton pair $\mathrm{f}^{+}, \mathrm{f}^{-}$is produced in the final state [see Eq. (2.3)]. We can distinguish between two cases. In the first case (Fig. 8), the lepton pair may consist of particles different from electrons and positrons (II). In the second case, we only have an electron-positron pair in the final state which implies that we have also to consider the production mechanism of Fig. 9 (III ,IV). Starting with process II (Fig. 8), we proceed in an analogous way as has been done before. The contribution to $\mathrm{d} \sigma_{\mathrm{ff}} / \mathrm{ds}$ ' can be split into three parts:

$$
\frac{d \sigma^{(2), \text { II }}}{d s^{\prime}}=\frac{1}{s} \sigma^{(0)}\left(s^{\prime}\right)\left[\delta\left(1-s^{\prime} / s\right)\left(\Delta_{2}^{s}\left(\varepsilon, m_{f}\right)+\Delta_{2}^{V}\left(m_{f}\right)\right)+\vartheta\left(1-s^{\prime} / s-\varepsilon\right) \Delta_{2}^{H}\left(s^{\prime} / s\right)\right]
$$


It is now natural to add the non-photonic virtual graph (Fig. 10) to the fermion paix production cross-section of Eq. (2.3). This is justified by the observation that, in general, the energy resolution $\Delta$ [see Eq. (2.13)] is much larger than the lepton mass $m_{f}$ (mostly $f=e^{ \pm}, \mu^{ \pm}$) so that we are in the same situation as has been discussed for soft photons. Therefore, the virtual contribution is needed to cancel the collinear state divergences which arise in the cross-section if the lepton pair is produced in the forward direction with respect to the incoming electron or positron. If $\Delta$ is of the same order as or smaller than $m_{f}$, one has to distinguish between the virtual contribution $\Delta_{2}^{V}$ on the one hand and the lepton pair production cross-section $\Delta_{2}^{f^{+}} f^{-}$on the other hand. In this case, s'can become larger than $s(1-2 \mathrm{~m} / \sqrt{\mathrm{s}})^{2}$ which means that the production of an additional. dilepton pair is inhibited, so that only $\Delta_{2}$ has to be added to the radiative correction. Therefore, Eq. (2.31) has to be modified and it reads

$\frac{d \sigma_{e^{+} e^{-}}^{(2), I I}}{d s^{\prime}}=\frac{1}{s} \sigma^{\left(s^{\prime}\right.}\left(s^{\prime}\right)\left[\delta\left(1-s^{\prime} / s\right) \Delta_{2}^{V}\left(m_{f}\right)+\vartheta\left(1-\frac{s}{s}-\frac{4 m_{f}}{v s}+\frac{4 m_{f}^{2}}{s}\right) \Delta_{2}^{f^{+} f^{\prime}}\left(s^{\prime} / s\right)\right]$

Notice that in Eq. (2.32) we are not allowed to neglect $4 \mathrm{~m}_{\mathrm{f}} / \sqrt{\mathrm{s}}$ with respect to $1-s^{\prime} / \mathrm{s}$ in $\Delta^{\mathrm{f}^{+} \mathrm{f}^{-}}$which could be done for $\Delta_{2}^{\mathrm{H}}$ in Eq. (2.31). Equation (2.32) is only relevant for heavy fermion pair production and we will limit ourselves in the subsequent discussion to Eq. (2.31). The virtual contribution to Eq. (2.31) is given by

$$
\Delta_{2}^{v}\left(m_{f}\right)=2 R_{\text {re }} \bar{T}_{\text {vac }}^{(2)}(s)
$$

where $\mathrm{F}_{\mathrm{vac}}^{(2)}(\mathrm{s})$ represents the virtual graph (Fig. 10) with the vacuum polarization insertion. For $s \gg \mathrm{m}_{\mathrm{f}}^{2}$ we have ${ }^{18)}$

$$
\operatorname{Re} F_{\text {vac }}^{(2)}(s)=\left(\frac{\alpha}{n}\right)^{2}\left[-\frac{1}{36} \ln ^{3} \frac{s}{m_{f}^{2}}+\frac{19}{72} \ln ^{2} \frac{s}{m_{f}^{2}}+\left(\frac{1}{3} 3(2)-\frac{26 s}{216}\right) \operatorname{m} \frac{s}{m_{f}^{2}}+D\left(\frac{m}{m_{f}}\right)\right]
$$

Here, we have assumed that $m_{f} \gg m . D\left(m / m_{f}\right)$ only depends on the masses:

$$
\begin{array}{ll}
m_{f}=m: D(1)=-\frac{11}{6} 3(2)+\frac{303}{100} \\
m_{f \rightarrow m} \quad: \quad D\left(\frac{m}{m_{f}}\right)=-\frac{1}{3} 3(3)-\frac{19}{103(2)}+\frac{3355}{1296}
\end{array}
$$

In order to calculate $\Delta_{2}^{S}$ and $\Delta_{2}^{H}$ we proceed as follows. The double differential cross-section for the process in Fig. 8 is given by 


$$
\begin{aligned}
& \frac{d \sigma_{e^{+} e^{-}}^{(2), \text { II }}}{d s^{\prime} d s^{\prime \prime}}=\sigma^{(0)}\left(s^{\prime}\right) \frac{1}{3 s^{\prime} s^{\prime \prime}} g^{1 / 2}\left(s_{,} s^{\prime}, s^{\prime \prime}\right) P\left(s^{\prime \prime}, m_{f}\right)\left(\frac{\alpha}{r}\right)^{2}[-2+ \\
& \left.\frac{\left(s_{-}-s^{\prime}-s^{\prime \prime}\right)^{2}+2 s^{\prime}}{g^{1 / 2}\left(s, s^{\prime}, s^{\prime \prime}\right)\left(s-s^{\prime}-s^{\prime \prime}\right)} \ln \left\{\frac{s^{\prime}-s^{\prime}-s^{\prime \prime}+g^{\prime \prime 2}\left(s_{,} s^{\prime}, s^{\prime \prime}\right)}{s_{-}-s^{\prime}-s^{\prime}-g^{\prime \prime 2}\left(s, s^{\prime}, s^{\prime \prime}\right)}\right\}\right]
\end{aligned}
$$

where $s^{\prime \prime}=\left(k_{1}+k_{2}\right)^{2}$ is the invariant mass of the lepton paix $f^{+}, f^{-}, P\left(s^{\prime \prime}, m_{f}\right)$ is given by Eq. (2.5) and $\mathrm{g}$ is defined by

$$
g(x, y, z)=x^{2}+y^{2}+z^{2}-2 x y-2 x z-2 y z
$$

$\Delta_{2}^{\mathrm{H}}$ can be easily obtained by integrating Eq. (2.36) over s"

$\Delta_{2}^{H}=\frac{s}{\sigma^{(a)}\left(s^{\prime}\right)} \int_{4 m_{F^{2}}^{2}}^{(\sqrt{3}-\sqrt{3},)^{2}} d s^{\prime \prime} \frac{d \sigma_{e^{\prime} e^{-2}}^{(2),}}{d s^{\prime} d s^{2}}=\left(\frac{\alpha}{n}\right)^{2}\left[\left\{\frac{1}{6} \frac{1+z^{2}}{1-z}\right\} \ln ^{2} \frac{s}{m_{F}^{2}}+\right.$

$\left\{\frac{1+z^{2}}{1-z}\left(\frac{2}{3} \ln (1-z)-\frac{1}{3} \ln z-\frac{5}{9}\right)-\frac{2}{3}(1-z)\right\} \ln \frac{5}{m_{f}^{2}}+\frac{1+z^{2}}{1-z}\left(\frac{2}{3} \ln ^{2}(1-z)-\frac{2}{3} \ln z \ln (1-z)+\right.$

$\left.\frac{1}{12} \ln ^{2} z-\frac{10}{9} \ln (1-z)+\frac{2}{9} \ln z-\frac{1}{6} h_{i 2}(1-z)-\frac{2}{3} 3(2)+\frac{28}{27}\right)-\frac{1}{6}(1-z)\left(\operatorname{hin}_{2}(1-z)+\right.$

$\left.\left.\frac{1}{2} \ln ^{2} z+8 \ln (1-z)-4 \ln z-2\right)-\frac{1}{3} z \ln z+\frac{16}{9}\right]$

The soft fermion contribution $\Delta_{2}^{S}$ is given by ${ }^{10)}$

$$
\begin{aligned}
& \Delta_{2}^{s}\left(\varepsilon, m_{f}\right)=\frac{s}{\sigma^{(1)}(s)} \int_{s(1-\varepsilon / 2)^{2}}^{s\left(1-\frac{2 m_{f}}{\sqrt{3}}\right)^{2}} \int_{4 m_{f}^{2}}^{\left(\sqrt{s}-\sqrt{s^{\prime}}\right)^{2}} d s^{\prime \prime} \frac{d \sigma_{e^{+} e^{*}}^{(2)}, I}{d s^{\prime} d s^{\prime \prime}}= \\
& =\frac{s}{\sigma^{(1 /}(s)} \int_{4 m_{f^{2}}^{2}}^{\varepsilon^{2} s / 4} d s^{\prime \prime} \int_{s(1-\varepsilon / 2)^{2}}^{\left(\sqrt{s}-\sqrt{s^{\prime}}\right)^{2}} d s^{\prime} \frac{d \sigma_{e^{+} e^{2}}^{(2), 2}}{d s^{\prime} d s^{\prime \prime}}=
\end{aligned}
$$




$$
\begin{aligned}
= & \left(\frac{\alpha}{n}\right)^{2}\left[\frac{1}{18} \ln ^{3} \frac{s}{m_{f}^{2}}-\frac{5}{18} \ln ^{2} \frac{s}{m_{f}^{2}}+\left(\frac{20}{27}-\frac{2}{3} 3(2)\right) \ln \frac{s}{m_{f}^{2}}+\frac{4}{3} 3(3)+\frac{10}{9} 3(2)\right. \\
& \left.-\frac{164}{31}+\frac{1}{3} \ln \varepsilon \ln \frac{s}{m_{f}^{2}}+\frac{2}{3} \ln ^{2} \varepsilon \ln \frac{s}{m_{f}^{2}}-\frac{10}{9} \ln \varepsilon \ln \frac{s}{m_{f}^{2}}+\frac{4}{9} \ln ^{3} \varepsilon-\frac{10}{9} \ln ^{2} \varepsilon+\left(\frac{56}{17}-\frac{4}{3} 3(2)\right) \ln \varepsilon\right]
\end{aligned}
$$

with $4 \mathrm{~m}_{\mathrm{f}} / \sqrt{\mathrm{s}} \ll \varepsilon \ll 1$. Hence it follows that for $s \gg \mathrm{m}_{\mathrm{f}}$ we can take $\sigma^{(0)}\left(\mathrm{s}^{\prime}\right)$ out of the integral since $\sigma^{(0)}\left(s^{\prime}\right) \approx \sigma^{(0)}(s)$. Collecting all contributions from the above equations we obtain

$$
\begin{aligned}
& \frac{d \sigma_{e^{+} e^{-}}^{(2), ~ I I ~}}{d s^{\prime}}=\frac{1}{3} \sigma^{(0)}\left(s^{\prime}\right)\left(\frac{\alpha}{n}\right)^{2}\left[\delta ( 1 - z ) \left[\left\{\frac{1}{3} \ln \varepsilon+\frac{1}{4}\right\} \operatorname{mn}^{2} \frac{s}{m_{f}^{2}}+\left\{\frac{2}{3} \ln ^{2} \varepsilon-\frac{10}{9}\right) \ln \varepsilon\right.\right. \\
& \left.\left.-\frac{17}{12}\right\} \ln \frac{s}{m_{f}^{2}}+\frac{4}{9} \ln ^{3} \varepsilon-\frac{10}{9} \ln ^{2} \varepsilon+\left(\frac{56}{27}-\frac{4}{3} 3(2)\right) \ln \varepsilon+D^{\prime}\left(\frac{m}{m_{f}}\right)\right] \\
& +\vartheta(1-z-\varepsilon)\left[\frac{1}{6} \frac{1+z^{2}}{1-z} \ln ^{2} \frac{s}{m_{f}^{2}}+\left\{\frac{1+z^{2}}{1-z}\left(\frac{2}{3} \ln (1-z)-\frac{1}{3} \ln z-\frac{5}{9}\right)-\frac{2}{3}(1-z)\right\} \ln \frac{s}{m_{f}^{2}}\right. \\
& +\frac{1+z^{2}}{1-z}\left(\frac{2}{3} \ln ^{2}(1-z)-\frac{2}{3} \ln z \ln (1-z)+\frac{1}{12} \ln ^{2} z-\frac{10}{9} \ln (1-z)+\frac{2}{9} \ln z-\frac{1}{6} \ln 2(1-z)\right. \\
& \left.\left.\left.-\frac{2}{3} 3(2)+\frac{20}{27}\right)-i(1-z)\left(\operatorname{li}_{2}(1-z)+\frac{1}{2} \ln ^{2} z+8 \ln (1-z)-4 \ln z-2\right)-\frac{1}{3} z \ln z+\frac{16}{9}\right]\right]
\end{aligned}
$$

with

$$
\begin{array}{ll}
m_{f}=m: & D^{\prime}(1)=\frac{4}{3} 3(3)-\frac{23}{9} 3(2)+\frac{921}{162} \\
m_{f} \gg m: & D^{\prime}\left(\frac{m}{m_{f}}\right)=\frac{2}{3} 3(3)-3(2)+\frac{227}{7^{2}}
\end{array}
$$

In the case of electron-positron pairs in the final state, we have to include the diagrams of Fig. 9 and the interference terms between Figs. 8 and 9 . The graphs in Fig. 9 have been calculated in the context of perturbative QCD in Ref. 14). For QED one has to omit the colour factor and the result equals 
$-15-$

$$
\begin{aligned}
& d \sigma_{e^{+} e^{-}}^{(2), \text { III }} \\
& \frac{d s^{\prime}}{d s^{\prime}}=\frac{1}{s} \sigma^{(0)}\left(s^{\prime}\right)\left(\frac{\alpha}{\pi}\right)^{2}\left[\left\{\frac{1}{2}(1+z) \ln z+\frac{1}{3 z}+\frac{1}{4}-\frac{1}{4} z-\frac{1}{3} z^{2}\right\} L^{2}+\right. \\
& \left\{(1+z)\left(2 \ln z \ln (1-z)-\ln ^{2} z+2 \operatorname{hi}(1-z)\right)+\left(\frac{4}{3 z}+1-z-\frac{4}{3} z^{2}\right) \ln (1-z)\right. \\
& \left.-\left(\frac{2}{3 z}+1-\frac{1}{2} z-\frac{4}{3} z^{2}\right) \ln z-\frac{8}{9 z}-\frac{8}{3}+\frac{8}{3} z+\frac{8}{9} z^{2}\right\} L+(1+z)\left(2 \ln z \ln ^{2}(1-z)-\right. \\
& \left.2 \ln ^{2} z \ln (1-z)+4 \ln (1-z) \operatorname{hin}_{2}(1-z)-5 \operatorname{hi} 2(-z)-5 \ln z \ln (1+z)\right)+\left(\frac{4}{3 z}+1-z-\frac{4}{3} z^{2}\right) \ln ^{2}(1-z) \\
& -\left(\frac{4}{3 z}+z-z-\frac{8}{3} z^{2}\right) \ln z \ln (1-z)-\left(\frac{16}{9} z+\frac{16}{3}-\frac{16}{3} z-\frac{16}{9} z^{2}\right) \ln (1-z)+\left(-\frac{4}{z}+2 z\right) S_{1,2}(1-z) \\
& +\left(-\frac{2}{z}-1-\frac{11}{2} z\right) \operatorname{hin}_{3}(1-z)+\left(\frac{10}{z}-10+3 z\right) \sin _{3}(-z)+\left(\frac{2}{z}+2+z\right)\left(6 S_{1,2}(-z)+\right. \\
& \left.6 \ln (1+z) \operatorname{lin}_{2}(-z)+3 \ln z \ln ^{2}(1+z)-\frac{5}{2} \ln ^{2} z \ln (1+z)+33(2) \ln (1+z)\right)+ \\
& \left(-5+\frac{1}{2} z\right) \ln z \lim _{2}(1-z)+\left(-\frac{10}{z}-4 z\right) \ln z \lim 2(-z)+\left(\frac{2}{3}+z\right) \ln ^{3} z+ \\
& \left(\frac{2}{3 z}-\frac{3}{2}+\frac{2}{3} z^{2}\right) \lim _{2}(1-z)+\left(\frac{1}{3 z}+\frac{3}{2}+\frac{9}{4} z-z^{2}\right) \ln ^{2} z+\left(\frac{8}{9 z}+\frac{51}{4}+\frac{1}{2} z-\frac{16}{5} z^{2}\right. \\
& \left.-\frac{3}{2} z 3(2)-63(2)\right) \ln z+\left(-\frac{2}{3 z}-3-2 z+\frac{2}{3} z^{2}\right) 3(2)+\left(\frac{6}{z}-9+\frac{3}{2} z\right) J(3) \\
& \left.+\left(\frac{44}{27 z}+\frac{147 z}{72}-\frac{385}{18} z-\frac{163}{216} z^{2}\right)\right]
\end{aligned}
$$

Finally, we have computed the interference terms originating from the graphs of Figs. 8 and 9. The calculation proceeds in an analogous way as has been done for the process above [see Ref. 14) and Appendix A]. Its contribution is given by the following expression

$$
\begin{aligned}
& \frac{d \sigma_{e^{+} e^{-}}^{(2), I I}}{d s^{\prime}}=\frac{1}{s} \sigma^{(0)}\left(s^{\prime}\right)\left(\frac{\alpha}{r 1}\right)^{2}\left[\left\{\frac{1+z^{2}}{1-z}\left(-\alpha i_{2}(1-z)-\frac{1}{2} \ln ^{2} z-\frac{3}{4} \ln z\right)-\right.\right. \\
& \left.\frac{7}{4}(1+z) \ln z-4+\frac{z}{2} z\right] L+2 \ln (1-z)\left(\frac{1+z^{2}}{1-z}(-\operatorname{hi} 2(1-z)-\right. \\
& \left.\left.\frac{1}{2} \ln ^{2} z-\frac{3}{4} \ln z\right)-\frac{7}{4}(1+z) \ln z-4+\frac{7}{2} z\right)+\frac{1+z^{2}}{1-z}\left(3 \lim _{3}(1-z)-8 S_{32}(1-z)+\right. \\
& 4 \lim _{3}(-z)+33(3)-2 \ln z \operatorname{hi}(-z)-6 \ln z \operatorname{hin}(1-z)+\frac{3}{2} 3(2) \ln z-\frac{1}{4} \ln ^{3} z+
\end{aligned}
$$




$$
\begin{aligned}
& \left.\frac{35}{12} \ln z-\frac{5}{4} \operatorname{lin}(1-z)+\frac{1}{2} \ln ^{2} z\right)+(1+z)\left(-\operatorname{li}_{3}(1-z)+\frac{1}{12} \ln ^{3} z-\right. \\
& \left.\frac{1}{2} \ln z \operatorname{li} 1(1-z)\right)+\left(2+\frac{1}{1-z}-\frac{2}{(1-z)^{2}}\right)(3(2)+2 \operatorname{li} 2(-z)+2 \ln z \ln (1+z))+ \\
& \frac{1}{(1+z)^{2}}\left(-183(2)-6 \ln ^{2} z-12 \lim (1-z)-24 \ln z+12\right)+\frac{1}{1+z}\left(363(2)+12 \ln ^{2} z+24 \operatorname{li} z(1-z)+\right. \\
& 7 \ln z-18)+\frac{12}{(1+z)^{3}} \ln z+\operatorname{li} 2(1-z)\left(-\frac{5}{4} z-\frac{z^{3}}{4}\right)-\frac{33}{2} 3(2)+\left(\frac{5}{2} z-8\right) \ln ^{2} z+ \\
& \left.\frac{1}{(1-z)^{2}} \ln z+\left(\frac{83}{12}-\frac{13}{12} z\right) \ln z-\frac{2}{(1-z)^{2}} \ln z-\frac{2}{1-z}+\frac{1495}{72}-\frac{905}{7^{2}} z-\frac{1}{9} z^{2}\right]
\end{aligned}
$$

Before finishing this section, we want to remark that the logarithmic mass singularities (collinear divergences) represented by the terms L [Eq. (2.13)] can be determined via renormalization group methods ${ }^{10)-12)}$. This we will show in Section 4 .

\section{3. - SOFT PHOTON RESUMMATION}

From the results obtained in the last section we infer that besides the large logarithms of the type $\mathrm{L}=\ln \left(\mathrm{s} / \mathrm{m}^{2}\right)$ the cross-sections will also be enhanced by the terms $\ln ^{i}(1-z) /(1-z)$ which become important near the boundary of phase space $z \rightarrow 1\left(s^{\prime} \rightarrow s\right)$. As will be discussed in section 5 , the latter terms constitute the bulk of the radiative corrections to the shape of the $z$ resonance which shows up at $z \sim 1$ for $/ s \approx M_{Z}$. These logarithmic enhanced terms only appear in process I [Eq. (2.30)] and process II [Eq. (2.40)] due to the emission of soft photons and soft fermion pairs respectively. Because this enhancement will show up in all orders one has to find a resummation technique in order to give an accurate prediction for the mass and width of the $Z$ boson.

In the case of process I these logarithms can easily be resummed $\left.\left.\left.{ }^{2}, 3\right), 10\right), 25\right)$ since they originate from the infra-red (IR) singularities appearing in the virtual [Eq. (2.24)] and real soft-photon [Eqs. (2.20) and $(2.26)$ ] cross-sections. According to Bloch-Nordsieck ${ }^{26)}$ and Yennie, Frautschi and Sura ${ }^{16)}$ these singularities exponentiate in QED in all orders of perturbation theory. As can be seen in Eq. (2.30) there exists a one-to-one correspondence between the $\ln ^{i}(1-z) /(1-z)$ and the $\ln ^{i+1} \varepsilon$ terms where the latter appear in the 
$\delta(1-z)$ part of the cross-section. Since these $\ln \varepsilon$ ( $\varepsilon$ is the soft-photon cut-off) are the remnants of the already cancelled IR singularities they have to exponentiate too. Therefore, Eqs. (2.19) or (2.30) can be generalized to all orders in the electromagnetiuc coupling constant so that it takes the form $\frac{d \sigma_{e^{+} e^{-}}^{I}}{d s^{\prime}}=\frac{1}{s} \sigma^{(0)}\left(s^{\prime}\right)\left[\delta(1-z) \delta^{V+s}\left(\alpha, \frac{s}{m^{2}}\right) \exp (\beta \ln \varepsilon)+\nu(1-z-\varepsilon) \delta^{H}\left(\alpha, z, \frac{s}{m^{2}}\right)\right]_{(3,1)}$

where $\delta^{\mathrm{V}+\mathrm{S}}$ and $\delta^{\mathrm{H}}$ represent the virtual/soft and hard photon contribution in all orders of perturbation theory. $\beta$ is defined by

$$
\beta=\frac{2 \alpha}{\pi}\left(\ln \frac{s}{m^{2}}-1\right)
$$

$\delta^{\mathrm{H}}$ can now be written as

$$
\delta^{H}\left(\alpha, z, \frac{s}{m^{2}}\right)=\sum_{n=1}^{\infty} \frac{1}{(n-1) !} \beta^{n} \frac{h^{n-1}(1-z)}{1-z} \delta^{V_{+}, s^{\prime}}\left(\alpha, \frac{s}{m^{2}}\right)+\tilde{\delta}^{H 1}\left(\alpha, z, \frac{s}{m^{2}}\right)
$$

Up to order $\alpha^{3}, \delta^{\mathrm{V}+\mathrm{S}}$ and $\tilde{\delta}^{\mathrm{H}}$ are given in Eq. (2.30). $\tilde{\delta}^{\mathrm{H}}$ is integrable in $z$ and the first term in Eq. (3.3) is wholly determined by the requirement that all the ln $\varepsilon$ terms originating from the integration over $z$ of $\delta^{H}$ are cancelled by the corresponding terms coming from $\delta^{\mathrm{V}+\mathrm{S}} \exp (\beta \ell n \varepsilon)$. Integrating expression (3.1) over the whole $x$ ange of $s^{\prime}=s z$ we obtain after some algebraic manipulation:

$$
\sigma_{e^{+} e^{-}}^{I}=\int_{s_{0}}^{s} d s^{\prime} \frac{d \sigma_{e^{+} e^{-}}^{I}}{d s^{\prime}}=\int_{z_{0}}^{1} d z \sigma^{(0)}(s z)\left\{\beta(1-z)^{\beta-1} \delta^{V+s}\left(\alpha, \frac{s}{m^{2}}\right)+\tilde{\delta}^{H-1}\left(\alpha, z, \frac{s}{m^{2}}\right)\right\}
$$

where in the above expression the $\ln (1-z)$ terms have been exponentiated. Equation (3.4) has also been derived in Ref. 10). The difference with their formulae is that we have included the full order $\alpha^{2}$ correction in $\delta^{\mathrm{V}+\mathrm{S}}$ and in $\tilde{\delta}^{\mathrm{H}}$. Furthermore, we agree with the authors of Ref. 10) that the soft-photon resummation as outlined above is very often incorrectly carried out in the iterature. One sees, e.g., that the term

$$
\beta(1-z)^{\beta-1} \delta^{v+s}\left(\alpha, s / m^{2}\right)
$$

in Eq. (3.4) has been replaced by

$$
\beta(1-z)^{\beta-1}+\left(\delta^{V+.5}\left(\alpha, \frac{s}{m^{2}}\right)-1\right)
$$


Likewise this has been done for the first term in Eq. (3.1) where

$$
\delta^{v+s}\left(\alpha, \frac{s}{m^{2}}\right) \exp (\beta \ln \varepsilon)
$$

is replaced by

$$
\exp (\beta \ln \varepsilon)+\left(\delta^{V+S}\left(\alpha, \frac{s}{m^{2}}\right)-1\right)
$$

Both replacements are wrong, since that would imply that all contributions like Eq. (2.24), where soft as well as virtual photons appear, will be neglected. This effect already shows up in order $\alpha^{2}$ which is clearly revealed by our explicit calculation in Eq. (2.30).

Notice that the resumation of the large terms $\ln ^{i}(1-z) /(1-z)$ is a non-perturbative one because expansion of expression (3.5) in $\alpha$ leads to a non-integrable integrand in Eq. (3.4), whereas the resummed expression is integrable. In contrast to process $I$ the $\ln ^{i}(1-z) /(1-z)$ terms appearing in process II [Eq. (2.40)] cannot so easily be resummed which is due to their different origin. In the latter case these terms originate from the final-state collinear divergence which arises if a lepton pair with small invariant mass is produced. Although these divergences cancel in $\mathrm{d} \sigma / \mathrm{ds}^{\prime}$ due to the KLN theorem ${ }^{19}$ ) they leave as a remnant the large logarithms originating from the upper part of the phase space integral in Eq. (2.39). However, the leading logarithms of process II can be determined in the following. For this purpose we rewrite Eq. $(2.40)$ as follows

$$
\begin{aligned}
\frac{d \sigma_{e^{t} e^{-}}^{\text {II }}}{d s^{\prime}} & =\frac{1}{s} \sigma^{(0)}\left(s^{\prime}\right)\left[\delta(1-z) \Delta\left(\alpha, \varepsilon, \frac{s}{m^{2}}\right)+\vartheta(1-z-\varepsilon)\left\{\frac{1}{1-z}\left(\frac{\alpha}{n}\right)^{2}(\right.\right. \\
& \left.\left.\left.\frac{1}{3} \ln ^{2} \frac{(1-z)^{2} s}{m_{f}^{2}}-\frac{10}{9} \ln \frac{(1-z)^{2} s}{m_{f}^{2}}-\frac{4}{3} 3(2)+\frac{56}{27}\right)+\ddot{\Delta}^{H}\left(\alpha, z, \frac{s}{m^{2}}\right)\right\}\right]
\end{aligned}
$$

where $\Delta^{\mathrm{V}+\mathrm{S}}$ and $\widetilde{\Delta}^{\mathrm{H}}$ are defined in the same way as below Eqs. (3.1) and (3.3). since the coefficients of the mass singular terms $L$ are known from the renormalization group functions (Section 4), we can also determine $\ln (1-z) /(1-z)$ except for $i=0$. Notice that this observation does not imply that these terms satisfy renormalization group equations like $L$ or exponentiate as in the case of Process $I$. This $c$ an be annoying since the leading $i$ in Process II (here $i=2$ ) is larger than the one in Process $I(i=1)$. However, in practice it turns out that these terms are harmless in soft fermion pair production since they are suppressed by large mass singular logarithms $L$ with respect to those appearing in 
Process I [compare Eq. (2.30) with (2.40)]. In the literature one has tried to improve expression (3.9). In Ref. 10), the authors include in Eq. (3.9) a11 initial-state photon radiations. Another approach has been followed in Ref. 12). Here one has observed that the large logarithms $L$ in the sum of processes $I$ and II satisfy an Altarel1i-Parisi equation 27). As has been shown by Gribov and Lipatov ${ }^{28)}$ this equation can be analytically solved for the leading logs $L$ in the 1 imit $z \rightarrow 1$. The result is that the first term in the curly brackets of Eq. (3.4) is replaced by

$$
\frac{\exp \left\{\frac{\eta}{2}\left(\frac{3}{2}-2 \gamma_{E}\right)\right\}}{\Gamma(\eta)}(1-z)^{\eta-1}
$$

with

$$
\eta=-6 \ln \left(1-\frac{\alpha}{3 \pi} L\right)
$$

Besides the large contributions near the boundary of phase space $(z \approx 1)$, there are also logarithmic enhanced terms of the type $\left(\ln ^{i} z\right) / z$ near $z \approx 0$. This is the region where the soft muon pairs dominate. These terms appear in Process III [Eq. (2.42)] and are due to the exchange of massless gauge bosons (here photons) between the electron and the positron line. They are therefore of coulombic origin.

If one wants to give an accurate prediction for soft muon pair production in higher order one has to make an attempt to resum these logarithmic enhanced terms. This has not been done yet. The only observation which can be made is that there exists a relation between the coefficients of these terms and the mass singular logarithms $\mathrm{L}$. This is akin to what we have seen for the large corrections near the boundary of phase space in Eq. (3.9). In the 1 imit $z \rightarrow 0$ $\left(s^{\prime} \rightarrow 0\right)$, Eq. $(2.42)$ can be written as

$$
\frac{d \sigma_{e^{+} e^{-}}^{\text {III }}}{d s^{\prime}} \underset{z \rightarrow 0}{=} \frac{1}{s} \sigma^{(0)}\left(s^{\prime}\right)\left(\frac{\alpha}{n}\right)^{2}\left[\frac{1}{3 z}\left\{\ln ^{2} \frac{s}{z m^{2}}-\frac{8}{3} \ln \frac{s}{z m^{2}}+\frac{44}{9}\right\}+\sigma\left(z^{\circ}\right)\right]
$$

We see that the lnz terms are completely absorbed by L. Since the coefficients of L can be obtained via the renormalization group (Section 4) one is also able to determine the coefficients of $\left(\ln ^{i} z\right) / z$. 
Before finishing this section, we want to state that the resummation of the large logarithms of the type $\ln ^{i}(1-z) /(1-z)$ already gives a reasonable estimate for the position of the $\mathrm{Z}$ peak. In this approximation, Eq. (3.4) becomes ${ }^{25)}$

$$
\sigma_{e^{+} e^{-}}^{I}=\int_{z_{0}}^{1} d z \sigma^{(0)}(s z) \beta(1-z)^{\beta-1}
$$

Furthermore we can approximate $\sigma^{(0)}(\mathrm{sz})$ in the neighbourhood of the $\mathrm{z}$-peak by the small width Breit-Wigner form

$$
\sigma^{(0)}\left(s^{\prime}\right)=\sigma^{(0)}\left(M_{z}^{2}\right) \frac{s^{\prime} \cdot \Gamma_{z}^{2}}{\left(s^{\prime}-M_{z}^{2}\right)^{2}+M_{z}^{2} \Gamma_{z}^{2}}
$$

Substitution of Eq. (3.14) into Eq. (3.13) yields, after keeping the most important terms 25 ),

$$
\sigma^{(I)}\left(M_{z}^{2}\right)=\sigma^{(0)}\left(M_{z}^{2}\right)\left(\frac{\Gamma_{z}}{M_{z}}\right)^{\beta}
$$

and the maximum of $\sigma_{e^{+} e^{-}}^{I}$ lies at ${ }^{13)}$

$$
\sqrt{s}=M_{z}+\frac{1}{8} \pi \beta \Gamma_{z}
$$

If we had only included the first-order correction, the position of the maximum would have been

$$
V_{s}=M_{z}+\frac{1}{\theta} \pi \beta \Gamma_{z}\left[1-\beta \ln \left(\frac{M_{z}}{\Gamma_{z}}\right)\right]^{-1}
$$

which overestimates the shift by almost a factor two. Finally we want to mention that one can very easily improve upon the small width approximation for the Z-boson propagator [see Eq. (3.14)] by including the Z-self energy correction via the replacement

$$
\frac{1}{s^{\prime}-M_{z}^{2}+i M_{z} \Gamma_{z}}
$$

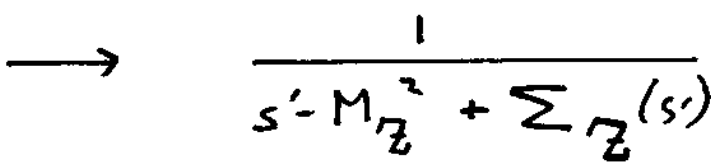




\section{4. - RENORMALIZATION GROUP METHOD}

As has already been mentioned in the previous sections, the coefficients of the large logarithms $\ln \left(s / \mathrm{m}^{2}\right)\left(s>>\mathrm{m}^{2}\right)$ can be obtained by using renormalization group methods. This is very interesting because it enables us to determine the dominant terms in radiative corrections without calculating the whole crosssection. Moreover it will serve as a check on our calculations. From Ref. 22) we know that these large logarithms represent the mass singularities (collinear divergences) which appear in integrated quantities like cross-sections or partial decay widths in the limit $m \rightarrow 0$. In our examples these singularities show up in the real as well as in the virtual photon graphs and their common origin can be traced back to the fact that one of the integrated momenta becomes collinear with an externa1 momentum.

The rules which determine these singularities have been derived in Ref. 22) and are applied to the parton cross-sections in the framework of perturbative QCD. Like the ultra-violet singularities, the initial-state collinear divergences satisfy a renormalization group equation provided they are factored out of the integrated quantities. This property of mass factorization which is well known in perturbative QCD also works for the mass singular terms in the cross-sections of QED. This observation has been made in Refs. 10)-12) and it was used to determine the leading $\ln \left(\mathrm{s} / \mathrm{m}^{2}\right)$ terms in the quantity $\mathrm{d} \sigma / \mathrm{ds} \mathrm{s}^{\prime}$. It is our goal to extend this work in order to find also the non-leading logarithms. Therefore we present a general formalism which enables us to calculate all mass singular terms in total cross-sections. It has been noticed in the last section that the process under investigation [Eqs. (2.2), (2.3)] can be described by the Drel1-Yan mechanism as far as the perturbative aspects are concerned. Mass factorization states that the Drell-Yan cross-section can be decomposed as follows 14 , 29)

$$
\begin{aligned}
& \frac{d \sigma_{i j}\left(s^{\prime}, s, m_{i}^{2}, m_{j}^{2}\right)}{d s^{\prime}}=\frac{1}{s} \sigma^{(0)}\left(s^{\prime}\right) \sum_{l, k} \int_{0}^{1} d x_{1} \int_{0}^{1} d x_{2} \int_{0}^{1} d x_{3} \delta\left(x_{1} x_{2} x_{3}-z\right) \\
& \Gamma_{l i}\left(x_{1}, \frac{\mu^{2}}{m_{i}^{2}}, g\left(\mu^{2}\right)\right) \Gamma_{k j}\left(x_{2}, \frac{\mu^{2}}{m_{j}^{2}}, g\left(\mu^{2}\right)\right) \tilde{\sigma}_{l k}\left(x_{3}, \frac{s^{\prime}}{\mu^{2}} \cdot g\left(\mu^{2}\right)\right)
\end{aligned}
$$

$m_{i}, m_{j}$ denote the masses of the incoming particles $i$ and $j$. In our case, $i=e^{-}$, $\mathrm{j}=\mathrm{e}^{+}$and $\mathrm{m}_{\mathrm{e}^{+}}=\mathrm{m}_{\mathrm{e}}=\mathrm{m}$. $\ell, \mathrm{k}$ represent all quanta of the underlying field theory which implies that in QED they are given by the $e^{ \pm}$and $\gamma \cdot g\left(\mu^{2}\right)$ is the electromagnetic coupling constant renormalized at $s^{\prime}=\mu^{2}$. $\Gamma_{l i}$ are the transition functions which contain all initial-state mass singularities represented by $\mathrm{m}_{\mathbf{i}}$. 
$\tilde{\sigma}_{\ell k}$ denotes the reduced cross-section which, in the QCD Ianguage, is very often called the DY Wilson coefficient. The most general form for $\Gamma_{\ell i}$ and $\tilde{\sigma}_{l k}$ is given by

$$
\begin{aligned}
& \Gamma_{l i}=\sum_{j=0}^{\infty} g\left(\mu^{2}\right)^{2 j}\left\{\sum_{n=0}^{j} a_{n j} \ln ^{n}\left(\frac{m^{2}}{\mu^{2}}\right)\right\} \\
& \tilde{\sigma}_{l k}=\sum_{j=0}^{\infty} g\left(\mu^{2}\right)^{2 j}\left\{\sum_{n=0}^{j} b_{n j} \ln ^{n}\left(\frac{s^{\prime}}{\mu^{2}}\right)\right\}
\end{aligned}
$$

From the coefficients $a_{n j}, b_{n j}$ in Eq. (4.2) and the mass factorization formula above, one can determine the $\ln \left(s / \mathrm{m}^{2}\right)$ terms in $\mathrm{d} \sigma / \mathrm{ds} \mathrm{s}^{\prime}$. This is because the $\ln \left(\mu^{2} / m_{i}^{2}\right)$ in the transition functions have to match the $\ln \left(s^{1} / \mu^{2}\right)$ terms appearing in $\widetilde{\sigma}_{\ell k}$ so that $d \sigma / d s^{\prime}$ becomes independent of $\mu^{2}$ after one has performed a finite coupling constant renormalization $g\left(\mu^{2}\right)=g(0) Z_{g}\left(\mu^{2} / m^{2}, g(0)\right)$.

In order to calculate the coefficients in the perturbation expansion of the above quantities we will use the convolution notation which is defined by

$$
(f \otimes g)(x)=\int_{0}^{1} d x_{1} \int_{0}^{1} d x_{2} \delta\left(x_{1} x_{2}-x\right) f\left(x_{1}\right) g\left(x_{2}\right)
$$

so that expression (4.1) can be written as

$$
\frac{d \sigma_{i j}}{d s^{\prime}}=\frac{1}{s} \sigma^{(0)}\left(s^{\prime}\right) \Gamma_{l i} \otimes \Gamma_{k j} \otimes \tilde{\sigma}_{l k}
$$

For our subsequent discussion it is also convenient to perform the Mel1in transform of Eq. (3.4)

$$
\frac{d \sigma_{i j}^{(n)}}{d s^{\prime}}=\frac{1}{s} \sigma^{(0)}\left(s^{\prime}\right) \Gamma_{l i}^{(n)} \Gamma_{k_{j}}^{(n)} \tilde{\sigma}_{l k}^{(n)}
$$

where the Mellin transform of a function $f(x)$ is given by

$$
f^{(n)}=\int_{0}^{1} d x x^{n-1} f(x)
$$

From mass factorization it follows that $\Gamma^{(n)}$ and $\tilde{\sigma}$ satisfy renormalization group equations 
$-23-$

$$
\begin{aligned}
& {\left[\left(\mu \frac{\partial}{\partial \rho}+\beta(g) \frac{\partial}{\partial g}\right)+\gamma_{a l}^{(n)}(g)\right] \Gamma_{l i}^{(n)}\left(\frac{\mu^{2}}{m_{i}^{2}}, g(\mu)\right)=0} \\
& {\left[\left(\mu \frac{\partial}{\partial \mu}+\beta(g) \frac{\partial}{\partial g}\right) \delta_{l a} \delta_{k b}-\gamma_{l a}^{(n)}(g) \delta_{k b}-\gamma_{k b}^{(n)}(g) \delta_{l a}\right] \tilde{\sigma}_{l k}\left(\frac{s^{\prime}}{\mu^{2}}, g(\mu)\right)=0}
\end{aligned}
$$

Equations (4.7) and (4.8) follow from Eq. (4.6) and the fact that $\sigma_{i j}^{(n)}$ is a renormalization group invariant

$$
\left[\mu \frac{\partial}{\partial \mu}+\beta(g) \frac{\partial}{\partial g}\right] \sigma_{i j}^{(n)}\left(\frac{s^{\prime}}{m^{2}}, g(\mu)\right)=0
$$

$\beta(g)$ is the beta function of QED. It is given by

$$
\beta(q)=-\beta \cdot \frac{g^{3}}{16 \pi^{2}}-\beta \cdot \frac{q^{5}}{\left(16 \pi^{2}\right)^{2}}+\cdots
$$

with $\beta_{0}=-4 / 3, \beta_{1}=4 \cdot \gamma_{a l}^{(n)}(g)$ are the matrix elements of the anomalous dimension matrix $\gamma^{(\mathrm{n})}(\mathrm{g})$ which will be denoted by

$$
\gamma^{(n)}(g)=\left(\begin{array}{cc}
\gamma_{\text {er }}^{(n)}(g) & \gamma^{(n)} \gamma e^{(g)} \\
\gamma_{e \gamma(n)}^{(n)} & \gamma^{(n)} \gamma \gamma(g)
\end{array}\right)
$$

with

$$
\gamma_{i j}^{(n)}(g)=\gamma_{0, i j}^{(n)} \frac{q^{2}}{16 \pi^{2}}+\gamma_{1, i j}^{(n)}\left(\frac{2^{2}}{16 n^{2}}\right)^{2}
$$

Subsequently we will also need the so-called splitting function $P_{i j}(z)$ which is given by

$$
\int_{0}^{1} d z z^{n-1} P_{i j}(z)=-\gamma_{i j}^{(n)}
$$

Further we need the coefficients of the non-logarithmic terms in the perturbation series of $\Gamma_{\ell i}$ and $\tilde{\sigma}_{\ell n}$ in Eq. (4.2). In order to make them more explicit we expand the latter quantities in the running coupling constant 


$$
\begin{aligned}
& \Gamma_{l i}^{(n)}\left(1, \bar{g}\left(m^{2}\right)\right)=\sum_{j=0}^{n} \Gamma_{j, l i}^{(n)} \bar{g}\left(m^{2}\right)^{2 j} \\
& \tilde{\sigma}_{l k}^{(n)}(1, \bar{g}(s))=\sum_{j=0}^{\infty} \tilde{\sigma}_{j, l k}^{(n)} \bar{g}\left(s^{\prime}\right)^{2 j}
\end{aligned}
$$

where $\bar{g}\left(s^{\prime}\right)$ up to lowest order in the $\beta$-function [Eq. (4.10)] is given by

$$
\frac{\bar{g}^{2}\left(s^{\prime}\right)}{16 \pi^{2}}=\frac{g^{2}\left(\mu^{2}\right) / 16 \pi^{2}}{1+\left(g^{2}\left(\mu^{2}\right) / 16 \pi^{2}\right) \beta_{0} \ln \left(\frac{s^{2}}{\mu^{2}}\right)}
$$

The coefficients $a_{n j}, b_{n j}$ which appear in the perturbation series of $\Gamma_{l i}$ and $\tilde{\sigma}_{l n}$ in Eq. (4.2) can now be inferred from the Callan-Symanzik equations in Eqs. (4.7) and (4.8) provided one knows the anomalous dimensions in Eq. (4.12) and the non-log coefficient $\Gamma_{j, l k}^{(n)}$ and $\widetilde{\sigma}_{j, l k}^{(n)}$ in Eqs. (4.14a,b). In practice it turns out that the coefficients in the perturbation series of $\tilde{\sigma}_{l k}$ cannot be directly calculated but are obtained from the quantities $\Gamma_{\ell i}$ and $d \sigma_{i j} / d s^{\prime}$. This means that we are only able to determine the $\ln \left(\mathrm{s} / \mathrm{m}^{2}\right)$ terms in $\mathrm{d} \sigma / \mathrm{ds}$ ' up to a given order in $g$ if the anomalous dimension is known in the same order. Furthermore, we need a complete knowledge of the coefficients in the perturbation expansion of $\mathrm{d} \sigma / \mathrm{ds}$ ' and $\Gamma_{\ell i}$ of the previous order. In our examples of the last section this implies that we have to know the lowest-order cross-section and splitting function in addition to the anomalous dimension in second order. The anomalous dimensions and the splitting functions are given by the Green's functions which correspond to the operator matrix elements of the composite operators $0_{\ell}^{(n)}(l=e, \gamma)$.

$$
\Gamma_{l i}^{(n)}\left(m_{i}^{2}\right)=\left\langle i\left|O_{l}^{(n)}(0)\right| i\right\rangle
$$

In QED $0_{\ell}^{(n)}$ stands for the electron/positron and photon operators which appear in the operator product expansion of the electroweak current correlation function in which the Drell-Yan cross-section ${ }^{30}$ ) is expressed.

Notice that the above identification [Eq. (4.16)] only holds if the mass factorization in Eq. (3.1) occurs at the point $s^{\prime}=\mu^{2}$. If we take another point, e.g., $s=\mu^{2}$, the $\Gamma_{\ell i}$ will not be given by the operator matrix element and its 
anomalous dimension except for the leading terms. The origin of this phenomenon can be attributed to the fact that the Fourier transform of the current correlation function is performed in $q$ momentum space $\left(s^{\prime}=q^{2}\right)$ and not in $p_{+}+p_{-}$ space $\left(s=\left(\mathrm{P}_{+}+\mathrm{p}_{-}\right)^{2}\right)$ [see also the remark in the footnote of Ref. 31)].

The anomalous dimensions for the composite operators in QED can be obtained from the literature where they have been calculated in the context of QCD provided one omits the colour factors. However, here arises another complication. In the literature, the expressions for the anomalous dimensions or the splitting functions $\mathrm{P}_{\mathrm{ij}}(\mathrm{z})$ [Eq. (3.26)] have been published in such a form that one cannot distinguish between transitions of the type $\gamma \rightarrow e^{+}$and $\gamma \rightarrow e^{-}$or $e^{-} \rightarrow e^{-}$and $e^{-} \rightarrow e^{+}$. In this case, Eq. (4.16) has to be interpreted as follows

$$
\begin{aligned}
& \left.\Gamma_{e^{-\gamma}}^{(n)}=\Gamma_{e^{+} \gamma}^{(n)}=\frac{1}{2}\left\langle\gamma\left|O_{e}^{(n)}(0)\right| y\right\rangle \text { (4.17a) }^{(n)}\right\rangle \Gamma_{e^{+} e^{-}}^{(n)}=\frac{1}{2}\left\langle e^{-}\left|O_{e^{(n)}(0)}^{(n)}\right| e^{-}\right\rangle \text {(4.17b) }_{e^{-}}^{(n)}=
\end{aligned}
$$

A further complication is that one has to disentangle the contributions of the two-photon and the fermion-antifermion final state to the operator matrix element if one wants to distinguish between photonic and non-photonic processes. This is only possible if one applies the cut vertex method ${ }^{32}$ to the calculation of the operator matrix element. Finally we want to remark that in QED a1l operators mix. This explains why we have to deal with a matrix [see Eq. (4.11)] rather than a real number.

Limiting ourselves to the examples in the previous section where the incoming particles are represented by $\mathrm{e}^{+} \mathrm{e}^{-}$, expression (4.1) can be written as

$$
\begin{aligned}
& \frac{d \sigma_{e^{+} e^{-}}^{(n)}}{d s^{\prime}}=\frac{1}{s} \sigma^{(0)}\left(s^{\prime}\right)\left[\Gamma_{e^{-}}^{(n)} \Gamma_{e^{+} e^{+}}^{(n)} \tilde{\sigma}_{e^{+} e^{-}}^{(n)}+\Gamma_{e^{+} e^{+}}^{(n)} \Gamma_{e^{-}}^{(n)} \tilde{\sigma}_{e^{+} \gamma}^{(n)}\right. \\
& \left.+\Gamma_{\gamma e^{-}}^{(n)} \Gamma_{e^{-e^{-}}}^{(n)} \tilde{\sigma}_{\gamma e^{-}}^{(n)}+\Gamma_{\gamma e^{+}}^{(n)} \Gamma_{\gamma e^{-}}^{(n)} \tilde{\sigma}_{\gamma \gamma}^{(n)}\right]
\end{aligned}
$$

Up to order $\alpha^{4}\left(\alpha=g^{2}(0) / 4 \pi\right)$ the last term in Eq. (4.18) does not contribute so that we only have to deal with the first three. From Eqs. (4.7), (4.8) and $(4.14), \Gamma_{l i}^{(n)}$ and $\tilde{\sigma}_{l k}^{(n)}$ can be expanded in perturbation theory as follows 
$-26-$

$$
\begin{aligned}
& \Gamma_{e e}^{(n)}=1+\frac{q^{2}}{16 \pi^{2}}\left[-\frac{1}{2} \gamma_{0, e e}^{(n)} \ln \frac{\mu^{2}}{m^{2}}+\Gamma_{0, e e}^{(n)}\right]+\frac{q^{4}}{\left(16 \pi^{2}\right)^{2}}\left[\left\{\frac{1}{6} \gamma_{0, e e}^{(n)}\left(\gamma_{0, e e}^{(n)}-2 \beta_{0}\right)+\right.\right. \\
& \left.\frac{1}{i} \gamma_{0, e \gamma}^{(n)} \gamma_{0, j e}^{(n)}\right\} \ln ^{2} \frac{\mu^{2}}{m^{2}}+\frac{1}{2}\left\{-\gamma_{1, e e}^{(n)}-2 \beta_{0} \Gamma_{0, e e}^{(n)}-\gamma_{0, e e}^{(n)} \Gamma_{0, e e}^{(n)}-\gamma_{0, e \gamma}^{(n)} \Gamma_{0, j e}^{(n)}\right\} \ln \frac{\mu^{2}}{m^{2}} \\
& +[1, \text { er }]
\end{aligned}
$$

$\Gamma_{\text {ea }}^{(n)}$ satisfies the equation [Eq. (4.7)]

$$
\left\{\mu \frac{\partial}{\partial \mu}+\beta(g) \frac{\partial}{\partial g}+\gamma_{e e}^{(n)}(g)\right\} \Gamma_{e e}^{(n)}+\gamma_{e \gamma}^{(n)} \Gamma_{\gamma e}^{(n)}=0
$$

with

$$
\Gamma_{\gamma e}^{(n)}=\frac{g^{2}}{16 \pi^{2}}\left[-\frac{1}{2} \gamma_{0, \gamma_{e}}^{(m)} \ln \frac{\mu^{2}}{m^{2}}+\Gamma_{0, \gamma_{e}}^{(m)}\right]
$$

In the same way $\tilde{\sigma}_{\mathrm{e}^{+} \mathrm{e}^{-}}^{(\mathrm{n})}$ is given by

$$
\begin{aligned}
& \tilde{\sigma}_{e^{+} e^{-}}^{(n)}=1+\frac{g^{2}}{16 \pi^{2}}\left[-\gamma_{0, e e}^{(n)} \ln \frac{s^{1}}{p^{2}}+\tilde{\sigma}_{0, e^{2} e^{-}}^{(n)}\right]+\frac{g^{4}}{\left(1, b r^{2}\right)^{2}}\left[\left\{\frac{1}{2} \gamma_{0, e e}^{(n)}\left(\gamma_{0, e e}^{(n)}-\beta_{0}\right)+\right.\right. \\
& \left.\frac{1}{4} \gamma_{0, e \gamma}^{(n)} \gamma_{0, \gamma e}^{(n)}\right\} \ln ^{2} \frac{s^{\prime}}{\mu^{2}}+\left\{-\gamma_{1, e e}^{(n)}-2 \beta_{0} \tilde{\sigma}_{0, e^{+} e^{-}}^{(n)}-\gamma_{0, e e}^{(n)} \tilde{\sigma}_{0, e+e}^{(n)}-\gamma_{0, y e}^{(n)} \tilde{\sigma}_{0, e y}^{(n)}\right\} \ln \frac{s^{\prime}}{\mu^{2}} \\
& \left.+\tilde{\sigma}_{1, e^{+e^{-}}}^{(n)}\right]
\end{aligned}
$$

which satisfies the equation

$$
\left\{r \frac{\partial}{\partial p}+\beta(g) \frac{\partial}{\partial g}-2 \gamma_{e e}^{(n)}(g)\right\} \tilde{\sigma}_{e^{+} e^{-}}^{(n)}-2 \gamma_{\gamma^{e}}^{(n)} \tilde{\sigma}_{e \gamma}^{(n)}=0
$$

with

$$
\sigma_{e^{+} \gamma}^{(n)}=\sigma_{e^{-} \gamma}^{(n)}=\frac{g^{2}}{16 \pi^{2}}\left[-\frac{1}{2} \gamma_{0,-\gamma}^{(n)} \ln \frac{s^{\prime}}{\mu^{2}}+\tilde{\sigma}_{0, e \gamma}^{(n)}\right]
$$

After having performed a finite coupling constant renormalization

$$
g^{2}\left(\mu^{2}\right)=g^{2}(0)\left(1-\frac{g^{2}(0)}{16 \pi^{2}} \beta_{0}\left(\ln \frac{\mu^{2}}{m^{2}}-\gamma_{E}+\ln (\pi)\right)\right.
$$


we can now substitute Eqs. (4.19) and (4.22) into Eq. (4.18). Taking the inverse Mellin transform we obtain

$$
\begin{aligned}
& \frac{d \sigma_{e^{+} e^{-}}}{d s^{\prime}}=\frac{1}{s} \sigma^{(0)}\left(s^{\prime}\right)\left[\delta(1-z)+\frac{\alpha}{4 \pi}\left[P_{0, e e}(z) \ln \frac{s^{\prime}}{m^{2}}+\tilde{\sigma}_{0, e^{t} e^{-}}(z)+2 \Gamma_{0, e e}(z)\right]+\frac{\alpha^{2}}{16 \pi^{2}}\left[\left\{\frac{1}{2}(\right.\right.\right. \\
& \left.\left.P_{0, e e} \otimes P_{0, e e}\right)(z)-\frac{1}{2} \beta_{0} P_{0, e e}(z)+\frac{1}{4}\left(P_{0, e y} \otimes P_{0, y e}\right)(z)\right\} \ln \frac{s^{\prime}}{m^{2}}+\left\{P_{\text {i,ee }}(z)-\beta_{0}\left(\tilde{\sigma}_{0, e t e}(z)-\left(\gamma_{\varepsilon}-\operatorname{hin}\right)\right.\right. \\
& \left.\left.P_{0, e c}(z)\right)+P_{0, e e} \otimes\left(\tilde{\sigma}_{0, e+e^{-}}+2 \Gamma_{0, e e}\right)(z)+\left(P_{0, e y} \otimes \Gamma_{0, y e}\right)(z)+\left(\tilde{\sigma}_{0, e y} \otimes P_{0, j e}\right)(z)\right] \ln \frac{s^{\prime}}{m^{2}}+ \\
& \beta_{0}(y-\ln 4 \pi)\left(\sigma_{0, e+e}(z)+2 \Gamma_{e e}^{(0)}(z)\right)+2 \Gamma_{1, e e}(z)+\tilde{\sigma}_{1, e+e}+2\left(\tilde{\sigma}_{0, e+e} \otimes \Gamma_{\text {oee }}\right)(z)+ \\
& \left.z\left(\tilde{\sigma}_{0, e \gamma} \otimes \Gamma_{\text {i,je }}\right)(z)\right]
\end{aligned}
$$

The splitting functions $P_{i j}(z)$ [see Eq. (4.13)] which appear in the above expressions have been calculated in the literature and can be found in Ref. 33).

According to the last section we split the cross-section into four parts which refer to the processes I-IV given in Eqs. (2.30), (2.40), (2.42) and $(2.43)$. Consequently we have also to split $P_{1, e e}(z)$ which is obtained from Eq. (16) in Ref. 33) into four parts.

Limiting ourselves to the logarithmic terms in second order only, we obtain:

Process I

$$
\begin{aligned}
& \frac{d \sigma_{e^{+} e^{-}}^{I}}{d s^{\prime}}=\frac{1}{s} \sigma^{\prime \alpha}\left(s^{\prime}\right)\left[\delta(1-z)+\frac{\alpha}{4 \pi}\left[P_{0, e e}(z) \ln \frac{s^{\prime}}{m^{2}}+\tilde{\sigma}_{0, e^{+} e^{-}}(z)+2 \Gamma_{0, e e}(z)\right]+\right. \\
& \frac{\alpha^{2}}{16 n^{2}}\left[\frac{1}{2}\left(P_{0, e e} \otimes P_{0, e e}\right)(z) \ln ^{2} \frac{s^{\prime}}{m^{2}}+\left\{P_{1, e e^{I}}(z)+\left(P_{0, e e^{\otimes}} \otimes\left(\tilde{\sigma}_{0, e e^{-}}+2 \Gamma_{0, e e}\right)\right)(z)\right\} \ln \frac{s^{\prime}}{m^{2}}\right]
\end{aligned}
$$$$
P_{0, e e}(z)=4\left\{\frac{1+z^{2}}{1-z}+\delta(1-z)\left(\frac{3}{2}+2 \ln \varepsilon\right)\right\} \quad(\text { fig. } 11)
$$

$$
\tilde{\sigma}_{0, e+e^{-}}(z)+2 \Gamma_{0, e e}(z)=4\left\{-\frac{1+z^{2}}{1-z}(1+\ln z)-\delta(1-z)(2-23(2)+2 \ln \varepsilon)\right\}
$$




$$
\begin{gathered}
\Gamma_{0, e e}(z)=2\left\{-\frac{1+z^{2}}{1-z}\left(\ln (1-z)+\frac{1}{2}\right)+\delta(1-z)(1-\ln \varepsilon)\right\}-\left(y_{z}-\ln 4 n\right) P_{0, e e}(z) \\
(j-11)
\end{gathered}
$$

$P_{1}^{I}$ eed $\left.z\right)=16\left[\frac{1+z^{2}}{1-z}\left\{-h z \ln (1-z)+\frac{1}{2} \operatorname{hin}^{2} z+h i_{2}(1-z)\right\}-\frac{1}{4}(1+z) \ln ^{2} z\right.$

$\left.+\ln z+3 / 2-z+\delta(1-z)\left(\frac{3}{16}-\frac{3}{2} 3(2)+33(3)\right)\right]$

$$
(f ; .12)
$$

Process II

$$
\begin{aligned}
& \frac{d \sigma_{e^{+} e^{-}}^{\pi}}{d s^{\prime}}=\frac{1}{s} \sigma^{(0)}\left(s^{\prime}\right) \frac{\alpha^{2}}{16 n^{2}}\left[-\frac{1}{2} \beta_{0} P_{0, e e}(z) \ln ^{2} \frac{s^{\prime}}{m^{2}}+\left\{P_{1, e e}^{I I}(z)-\right.\right. \\
& \left.\beta_{0}\left(\tilde{\sigma}_{0, e^{+} e^{-}}(z)-\left(y_{E}-\ln (n) P_{0, e e}(z)\right)\right\} \ln \frac{s^{\prime}}{m^{2}}\right] \\
& P_{1, e e}(z)=16\left[\frac{1+z^{2}}{1-z}\left(-\frac{1}{3} \ln z-\frac{5}{9}\right)-\frac{2}{3}(1-z)-\delta(1-z)\left(\frac{1}{12}+\right.\right. \\
& \left.\left.\frac{2}{3} \zeta(2)+\frac{10}{9} \ln \varepsilon\right)\right] \quad(j i j \cdot 13)
\end{aligned}
$$

$\frac{\text { Process III }}{d \sigma^{\text {III. }}}$

$$
\begin{aligned}
& \frac{d \sigma^{\frac{I I}{e^{+} e^{-}}}}{d s^{\prime}}=\frac{1}{s} \sigma^{(0)}\left(s^{\prime}\right) \frac{\alpha^{2}}{16 \pi^{2}}\left[\frac{1}{4}\left(P_{0, e \gamma} \otimes P_{0, \gamma e}\right)(z) \ln \frac{2 s^{\prime}}{m^{2}}+\right. \\
& \left.\left\{P_{1, e e}^{I I I}(z)+\left(P_{0, e y} \otimes \Gamma_{0, \gamma^{e}}\right)(z)+\left(\tilde{\sigma}_{0, e y} \otimes P_{0, y e}\right)(z)\right\} \ln \frac{s^{\prime}}{m^{z}}\right] \\
& P_{0, e p}(z)=4\left\{z^{2}+(1-z)^{2}\right\} \quad(\text { jig.14 }) \\
& P_{0, y e}(z)=4\left\{\frac{1+(1-z)^{2}}{z}\right\} \quad(\text { fij.15 }) \\
& \Gamma_{0, \gamma e}(z)=-\frac{1}{2} P_{0, \gamma e}(z)(2 \ln z+1) \quad(\text { fig. 15) } \\
& \tilde{\sigma}_{0, e y}(z)=\frac{1}{2} P_{0, e y}(z) \ln \frac{(1-z)^{2}}{z}+1+6 z-7 z^{2}(\text { jy.16) }
\end{aligned}
$$




$$
\begin{gathered}
P_{1, e e}^{\text {III }}(z)=16\left\{-\frac{1}{2}(1+z) \ln ^{2} z+\left(\frac{1}{2}+\frac{5}{2} z+\frac{4}{3} z^{2}\right) \ln z+\frac{1-z}{2 z}\left(\frac{20}{9}+\frac{2}{9} z+\frac{56}{9} z^{2}\right)\right\} \\
(\text { fig. 17) }
\end{gathered}
$$

Process IV

$$
\begin{aligned}
& \frac{d \sigma_{e^{+} e^{-}}^{\text {II }}}{d s^{\prime}}=\frac{1}{s} \sigma^{(0)}\left(s^{\prime}\right) \frac{\alpha^{2}}{16 \pi^{2}}\left[P_{1, e e}^{\text {II }}(z) \ln \frac{s^{\prime}}{m^{2}}\right] \\
& P_{1, e e}^{\text {II }}(z)=16\left\{\frac{1+z^{2}}{1-z}\left(-\frac{1}{2} \ln ^{2} z-\operatorname{li} 2(1-z)-\frac{3}{4} \ln z\right)-\frac{7}{4}(1+z) \ln z\right. \\
& \left.-4+\frac{7}{2} z\right\} \quad(j ; .18)^{(4.34)}
\end{aligned}
$$

Substitution of the renormalization coefficients $P_{i j}, \Gamma_{i j}$ and $\tilde{\sigma}_{i j}$ in the expression for the cross-sections reveals that our results for the leading and next-to-leading logarithms are in complete agreement with the exactly calculated ones in section 2 .

\section{5. - DISCUSSION AND NUMERICAL RESULTS}

Here we will discuss the results of our calculations which have been derived in the previous sections. In particular the discussion will be focused on the application of the radiatively-corrected cross-sections to the forthcoming experiments carried out at SLAC (SLC) and CERN (LEP).

The first topic of our study is the behaviour of the differential crosssection $\mathrm{ds} / \mathrm{ds}^{\prime}$ at $/ \mathrm{s}=100 \mathrm{GeV}$.

In Fig. 19 we have plotted $\mathrm{d} \sigma / \mathrm{ds}$ ' which corresponds to the photonic (I) and non-photonic contributions (II,III+IV). In this and the subsequent figures, the $Z$-parameters have been taken as follows. $M_{Z}=93 \mathrm{GeV}, \Gamma_{Z}=2.5 \mathrm{GeV}$ and $\sin ^{2} \theta=0.223$. From this figure we infer that except for $s^{\prime} \ll s(z \rightarrow 0) d \sigma / d s^{\prime}$ is dominated by Process I. In particular in the region where the emitted photons become soft, the cross-section will be dominated by the infra-red singular terms: $\ln ^{i}(1-z) /(1-z)$ appearing in Eq. (2.30). For $z \rightarrow 1$ the next-to-1eading process is given by II since the same type of terms also appears here [see Eq. (2.40)]. In 
the latter case they are due to the emission of a soft additional lepton pair. Notice that in this process the effects of these terms are suppressed since they show up in higher order in $\alpha$. On the other hand, processes III and IV are completely suppressed in this region and they approach zero for $s^{\prime} \rightarrow s$. This situation changes if the muon-pair mass $s^{\prime}$ gets very small ( $\left.z \rightarrow 0\right)$. Here Process III dominates the three others, due to the exchange of a massless particle (photon) in the production mechanism of an additional lepton paix (Fig. 9). This is revealed by the Coulomb singular terms of the type $\ln ^{i} z / z$ in Eq. (2.42).

The last type of terms also shows up in the multiperipheral two-photon process given in Fig. 2034), which is also a QED process of the same order in $\alpha$. This process has not been considered so far, since it is a $\mu$-pair production by two spin-1 particles, whereas the $\mu$-pairs up to now were produced by a single vector particle. However, experimentally there is no a priori reason to exclude this production mechanism. The cross-section has been obtained by using the event generator programe of Ref. 35). Although dominating one-photon exchange for $z<0.3$, we see that the two-photon process does not give any contribution to the $\mathrm{Z}$-shape. From the total cross-section at $V \mathrm{~s}=100 \mathrm{GeV}$ which is equal to $203 \mathrm{nb}$, only remains $0.13 \mathrm{nb}$ if we put a $10 \mathrm{GeV}$ lower bound on $\sqrt{ } \mathrm{s}^{\prime}$. Therefore if one imposes realistic cuts on the muon pair mass $s^{\prime}$, the two-photon contribution turns out to be unimportant for $\mathrm{Z}$-production. Moreover, one has to bear in mind that the largest part of the muons vanishes in the beam pipe.

Summarizing our findings we conclude that for small s' the Processes II, III and IV are completely overwhelmed by the two-photon reaction. For large $s^{\prime}$ where the Z-peak appears, Reaction I dominates all the others, so that in the rest of this section we shall only limit ourselves to the latter one.

Before continuing our discussion how the Z-resonance shape is influenced by Process I, we want to give the results for the total cross-section due to the $\gamma^{*}$ exchange (Fig. 1), since this part can be analytically calculated. $\sigma_{\text {tot }}(s)$ is given by

$$
\sigma_{\text {tat }}(s)=\int_{s_{0}}^{s} d s^{\prime} \frac{d \sigma}{d s^{\prime}}
$$

where so denotes the threshold $s_{0}=4 \mathrm{~m}_{\mu}^{2}$. From the expressions in Section 2 we infer that the bulk of the contribution to $\sigma_{\text {tot }}$ is coming from the threshold region where $d \sigma / d s^{\prime}$ diverges as $\ln ^{i} z / z^{j}(j=0$ or 1$)$ for $z \rightarrow 0$. The results are 


$$
\begin{aligned}
& \sigma_{\text {tot }}^{\gamma^{*} I}(s)=\frac{4 \pi \alpha^{2}}{35}\left[1+\frac{\alpha}{\pi}\left[\left\{L_{\mu}-\frac{7}{6}\right\} L-L_{\mu}+23(2)+\frac{2}{3}\right]+\left(\frac{\alpha}{\pi}\right)^{2}\left[\left\{\frac{1}{4} L_{\mu}^{2}\right.\right.\right. \\
& \left.-\frac{1}{3} L_{\mu}-\frac{5}{2} 3(2)+\frac{133}{72}\right\} L^{2}+\left\{-\frac{1}{12} L_{\mu}^{3}-\frac{5}{6} L_{\mu}{ }^{2}+\left(7 / 23(2)+\frac{47}{18}\right) L_{\mu}+33(3)-\frac{77}{6} 3(2)\right. \\
& \left.-\frac{3047}{432}\right\} L+\frac{1}{24} L_{\mu}^{4}+\frac{1}{18} L_{\mu}^{3}+\left(-\frac{5}{4} 3(2)+\frac{7}{18}\right) L_{\mu}{ }^{2}+\left(\frac{3}{2} 3(3)-\frac{7}{3} 3(2)\right. \\
& \left.\left.\left.-\frac{1}{54}\right) L_{\mu}-\frac{7}{20} 3(2)^{2}-83(3)+\frac{1051}{7^{2}} 3(2)-63(2) \ln 2+\frac{517}{324}\right]\right]
\end{aligned}
$$

$$
\begin{aligned}
& \text { where } L_{\mu}=\ln \left(s / m_{\mu}^{2}\right) . \\
& \sigma_{\text {tot }}^{\gamma^{*}-\pi}(s)=\frac{4 \pi \alpha^{2}}{3 s}\left(\frac{\alpha}{n}\right)^{2}\left[\left\{\frac{1}{6} L_{\mu}-\frac{7}{36}\right\} L^{2}+\left\{\frac{1}{6} L_{\mu}^{2}-\frac{16}{9} L_{\mu}-\frac{1}{3} 3(2)\right.\right. \\
& \left.+\frac{347}{108}\right\} L-\frac{4}{9} L_{\mu}^{2}+\left(\frac{125}{27}-3(2)\right) L_{\mu}+D^{\prime \prime}\left(\frac{m}{m_{f}}\right)
\end{aligned}
$$

with

$$
m_{f}=m \quad D^{\prime \prime}(1)=\frac{4}{3} 3(3)+\frac{z}{3} \zeta(2)-\frac{9^{51}}{162}
$$

$$
m_{f} \gg m \quad D^{\prime \prime}\left(\frac{m}{m_{f}}\right)=\frac{2}{3} 3(3)+\frac{35}{9} 3(2)-\frac{5045}{648}
$$

$$
\begin{aligned}
& \sigma_{\text {tot }}^{\gamma^{*}, \text { III }}(s)=\frac{4 \pi \alpha^{2}}{35}\left(\frac{\alpha}{\pi}\right)^{2}\left[\frac{s}{15 m_{\mu}^{2}}\left[L^{2}+\left\{2 L_{\mu}-\frac{117}{15}\right\} L+L_{\mu}^{2}-\frac{117}{15} L_{\mu}-23 / 2\right)\right. \\
& \left.+\frac{5102}{225}\right]+\left\{-\frac{1}{4} L_{\mu}^{2}+\frac{13}{12} L_{\mu}+\frac{1}{2} 3(2)-\frac{83}{36}\right\} L^{2}+\left\{-\frac{1}{3} L_{\mu}^{3}+\frac{13}{6} L_{\mu}^{2}+\left(23(2)-\frac{107}{9}\right) L_{\mu}\right. \\
& \left.-43(3)-\frac{23}{3} 3(2)+\frac{634}{27}\right\} L_{-}-\frac{1}{6} L_{\mu}+\frac{11}{18} L_{\mu}^{3}+\left(\frac{15}{2} 3(2)-\frac{1015}{7^{2}}\right) L_{\mu}^{2}+ \\
& \left.\left(-183(3)-\frac{37}{2} 3(2)+\frac{14077}{216}\right) L_{\mu}-\frac{57}{5} 3(2)^{2}+173(3)+\frac{877}{12} 3(2)-\frac{202999}{1296}\right]
\end{aligned}
$$

The leading part of expression (5.5) $\left(\sim \mathrm{s} / \mathrm{m}_{\mu}^{2}\right)$ has been also given in Eq. (4.3) of Ref. 36). Notice that our result differs from theirs as far as the coefficients of the non-log terms $L$ are concerned. 


$$
\begin{aligned}
& \sigma_{\text {tot }}^{\gamma^{*}, \mathbb{R}}(s)=\frac{4 \pi \alpha^{2}}{3 s}\left(\frac{\alpha}{\pi}\right)^{2}\left[\left\{-\frac{1}{6} L_{\mu}^{3}+\frac{25}{12} L_{\mu}^{2}-\frac{203}{18} L_{\mu}-43(3)+\frac{1351}{54}\right\} L\right. \\
& +\frac{1}{24} L_{\mu}^{4}-\frac{4}{9} L_{\mu}^{3}+\left(23(2)+\frac{35}{36}\right) L_{\mu}^{2}+\left(-3(3)-\frac{32}{3} \zeta(2)+\frac{1793}{216}\right) L \nu \\
& \left.+\frac{22}{10} 3(2)^{2}-\frac{1}{12} 3(3)+\frac{731}{10} 3(2)-333(2) \ln 2-\frac{7005}{324}\right]
\end{aligned}
$$

The $\sigma_{\text {tot }}(s)$ for the two-photon process in Fig. 20 can be found in Eq. (36) of Ref. 34) and is therefore not given here.

As has already been mentioned, we shall now concentrate on the photonic contribution to the shape of the z-resonance. The first object of our study is the behaviour of the quantity

$$
W\left(s^{\prime}\right)=\frac{s}{\sigma^{(0)}\left(s^{\prime}\right)} \frac{d \sigma}{d s^{\prime}}
$$

which accounts for the photonic radiative correction to the Born graph in Fig. 1. Note that, by definition, $W\left(s^{\prime}\right)$ does not depend on $M_{Z}$ and $\Gamma_{Z}$. In Fig. 21 we have presented the order $\alpha$ contribution to W( $\left.\mathrm{s}^{\prime}\right)$ due to Eq. (2.11) (dashed line) at $\sqrt{s}=100 \mathrm{GeV}$. In the same figure we have also given $\mathrm{W}\left(\mathrm{s}^{\prime}\right)$ corrected up to order $\alpha^{2}$ [Eq. (2.30)] (solid line) where the soft photons have been resummed according to Eq. (3.4). If we had plotted Eq. (2.30) in the same range without resummation, the difference would not have been noticeable.

The differences between the $O(\alpha)$ and $O\left(\alpha^{2}\right)$ radiative corrections become more clear if we study their effect on the total cross-section given in Eq. (5.1). This is shown in Fig. 22 where the total cross-section is given for $50<\sqrt{s}<200 \mathrm{GeV}$. Here we compare the Born approximation with the $O(\alpha)$ and $0\left(\alpha^{2}\right)$ corrected cross-sections.

The resonance peak is distorted due to the large enhanced logarithms $\ln ^{i}(1-z) /(1-z)$ of Process I (see Section 3). This effect is due to soft-photon radiation. Remark that the tilt of the right-hand side of the peak is only due to these terms and not to the large "mass singular" logarithms $\ln \left(\mathrm{s} / \mathrm{m}^{2}\right)$. The latter only determine the size of the effect. Notice that this effect is reduced by the inclusion of the second-order radiative correction. 
In Fig. 23, the top of the $z$-peak is given in more detail. Here the zeroth order cross-section lies outside the frame of the figure. The position of the peak is shifted with respect to the Born cross-section by about $200 \mathrm{MeV}$ in the $0(\alpha)$ corrected approximation. If we include soft-photon resummation, this shift is reduced to about $100 \mathrm{MeV}$. The same happens if we include the $0\left(\alpha^{2}\right)$ correction with and without soft-photon resummation. The precise values for the shift and the height of the peak can be found in the Table.

From the above observation we infer that the inclusion of soft photons in all orders of perturbation theory improves the results obtained from the $O(\alpha)$ [Eq. (2.11)] as well as $0\left(\alpha^{2}\right)$ [Eq. (2.30)] approximation and that the best result is given by the $0\left(\alpha^{2}\right)$ soft-photon improved cross-section in Eq. (3.4). We conclude that the maximum of the peak is shift upwards by an amount of $128 \pm 5 \mathrm{MeV}$, and that the peak value is reduced to $1.38 \pm 0.01 \mathrm{nb}$.

We have checked that soft-fermion paix production in Process II, Eq. (2.40) (a11 fermion species together) can at most change the position of the peak by 1 or $2 \mathrm{MeV}$.

In Fig. 24 we compare our best result with the two soft-photon approximations given by Eqs. (3.10) and (3.13). Expression (3.10) is in good agreement with the $0\left(\alpha^{2}\right)$ soft-photon improved result. The latter also contains the complete hard-photon radiation so that we can conclude that its influence is very small at the $\mathrm{z}$-peak. This situation will change if we go to energies considerably higher than $M_{Z}$. For example at $\sqrt{ }=110 \mathrm{GeV}$, Eqs. (3.10) and (3.13) deviate from the $0\left(\alpha^{2}\right)$ soft-photon improved result by about 30 and 15 per cent.

Summarizing our results, we have calculated in two independent ways analytic formulae for the $O\left(\alpha^{2}\right)$ QED initial state corrections to the differential crosssection $d \sigma / d s^{\prime}\left(e^{+} e^{-\rightarrow \mu^{+}} \mu^{-}\right)$. We found that they are completely dominated by the photonic contributions except for low $s^{\prime}$ where anyhow the two-photon crosssection is much larger than the annihilation one. The shift of the $Z$-peak due to these corrections has been determined with an accuracy which is more than sufficient for LEP experiments. 


\begin{tabular}{|l|c|c|}
\hline & $\begin{array}{c}\text { height } \\
\text { (nb) }\end{array}$ & $\begin{array}{c}\text { position } \\
\text { (Gev) }\end{array}$ \\
\hline lowest order csx & 1.86 & 93.018 \\
estimates from Eqs. (3.15) and (3.16) & 1.3 & 93.11 \\
soft-photon approximation, Eq. (3.13) & 1.29 & 93.128 \\
soft-photon approximation, Eq. (3.10) & 1.36 & 93.135 \\
$0(\alpha)$ corrected csx, Eq. (2.11) & 1.32 & 93.205 \\
$0(\alpha)$ corrected csx + soft-photon resummation, \\
Eqs. (2.11) and (3.4) \\
$0\left(\alpha^{2}\right)$ corrected csx, Eq. (2.30) & 1.39 & 93.127 \\
$0\left(\alpha^{2}\right)$ corrected csx + soft-photon resummation, \\
Eqs. (2.30) and (3.4)
\end{tabular}

TABLE - Comparison of the various estimates for the change of height and position of the Z-peak due to initial-state photonic corrections for $M_{Z}=93 \mathrm{GeV}, \Gamma_{Z}=2.5 \mathrm{GeV}$ and $\sin ^{2} \theta_{\mathrm{W}}=0.223$. 


\section{APPENDIX A}

In this Appendix we will give an outline of the calculation of the process in Eq. (2.2). Starting from Eq. (2.4) we have to evaluate the three-body phase space integral. For that purpose we go into the centre-of-mass frame of the incoming $\mathrm{e}^{+} \mathrm{e}^{-}$pair where the momenta are parametrized as follows.

$$
\begin{aligned}
& P_{+}=(E, 0,0,|\vec{p}|) \quad P_{-}=\left(E, 0,0,-\mid \overrightarrow{p_{1}}\right) \\
& k_{1}=\omega_{1}(1,-\sin \theta, 0, \cos \theta) \\
& k_{2}=\omega_{2}(1, \cos \theta \cos \varphi \sin x-\sin \theta \cos \chi, \sin \varphi \sin x, \cos \theta \cos x+\sin \theta \sin x \cos \varphi) \\
& (A .3)
\end{aligned}
$$

where $\chi$ is the angle between $\vec{k}_{1}$ and $\vec{k}_{2}$. Further we define the invariants

$$
s=\left(p_{+}+p_{-}\right)^{2} \quad s^{\prime}=\left(q_{+}+q_{-}\right)^{2}=q^{2} \quad s_{1}=\left(k_{1}+k_{2}\right)^{2} s_{3}=\left(k_{2}+q\right)^{2} \quad s_{4}=\left(k_{1}+q\right)^{2} \quad(\text { A.4) }
$$

$\omega_{i}, E$ and $|\vec{p}|$ can be expressed in these invariants as follows:

$$
E=\frac{1}{2} \sqrt{s} \quad|\beta|=\frac{1}{2} \sqrt{s-4 m^{2}} \quad \omega_{1}=\frac{s-s_{3}}{2 \sqrt{s}} \quad \omega_{2}=\frac{s-s_{4}}{2 \sqrt{s}}
$$

From the relations

$$
\cos x=1-\frac{2 s_{1}}{\left(s-s_{3}\right)\left(s-s_{4}\right)} \text { and } s+s^{\prime}=s_{1}+s_{3}+s_{4}
$$

we derive the boundaries of the Dalitz plot given in Fig. 7. Notice that in the above expressions the photons $k_{1}$ and $k_{2}$ are massless which will lead to the well-known infra-red (IR) divergences in the phase space integral of Eq. (2.4). The treatment of these IR divergences is a standard procedure in QED and it will be discussed in Appendix $B$.

In this Appendix we will limit ourselves to the hard-photon integral where the photon energies $\omega_{i}$ [Eq. (A.5)] are cut off from below, i.e., 


$$
\omega_{i} \geqslant \Delta=\frac{1}{2} \varepsilon \sqrt{5} \text { inth } \Delta \ll m
$$

In our calculation we have checked that in the final result $\Delta$ can also be taken larger than $m$ without losing finite terms (see Appendix $B$ ). The integration over the phase space will then be modified as is indicated in Fig. 7. Integrating over the remaining variables, Eq. (2.4) becomes

$$
\frac{d \sigma}{d s^{\prime}}=\sigma^{(0)}(s) \frac{1}{64(2 \pi)^{5} s} \int d s_{3} d s_{4} d \Omega\left|T^{(2)}\right|_{(\mathrm{A} .8)}^{2}
$$

where $\mathrm{d} \Omega$ denotes the solid angle, i.e., $\mathrm{d} \Omega=\mathrm{d} \cos \theta \mathrm{d} \phi$ and the boundaries of $\mathrm{s}_{3}, \mathrm{~s}_{4}$ are given by (symmetric integration)

$$
\begin{array}{ll}
s^{\prime}+\varepsilon s<s_{3}<(1-\varepsilon) s & \frac{s s^{\prime}}{s_{3}}<s_{4}<s+s^{\prime}-s_{3} \\
s^{\prime}(1+\varepsilon)<s_{3}<s^{\prime}+\varepsilon s & \frac{s s^{\prime}}{s_{3}}<s_{4}<(1-\varepsilon) s
\end{array}
$$

The matrix element $\left|\mathrm{T}^{(2)}\right|^{2}$ has been computed by using the algebraic manipulation program REDUCE ${ }^{37)}$. Using the invariants defined in eq. (A.4) $\mid \mathrm{T}^{(2)} \mathrm{I}^{2}$ can be expressed as a sum of the following type of terms

$$
\left|T^{(2)}\right|^{2}=\sum \frac{N\left(D_{k}, s_{i}\right)}{D_{i_{1}} D_{i_{2}} D_{i_{3}} D_{i_{4}}}
$$

with $D_{k} \neq D_{i_{n}} . D_{i_{n}}$ are the denominators which originate from the propagators appearing in the Feynman graphs of Fig. 4. Hence it follows that the maximum number of factors $D_{i_{n}}$, which can be equal, does not exceed four. The possible $D_{i_{n}}$ which can appear are given by

$$
\begin{aligned}
& D_{1}=\left(p_{4}-k_{1}\right)^{2}-m^{2} \quad D_{2}=\left(p_{-}-k_{2}\right)^{2}-m^{2} \quad D_{3}=\left(p_{-}-q\right)^{2}-m^{2} \quad D_{4}=\left(k_{1}+q\right)^{2}-m^{2} \\
& D_{5}=\left(p_{4}-q\right)^{2}-m^{2} \quad D_{6}=\left(k_{2}+q\right)^{2}-m^{2} \quad D_{7}=\left(p_{4}-k_{2}\right)^{2}-m^{2} \quad D_{8}=\left(p_{-}-k_{1}\right)^{2}-m^{2}
\end{aligned}
$$

From Eqs. (A.4) and (A.8), we conclude that only $\mathrm{D}_{1}, \mathrm{D}_{2}, \mathrm{D}_{3}, \mathrm{D}_{5}, \mathrm{D}_{7}$ and $\mathrm{D}_{8}$ explicitly depend on the angular variables $\theta$, and $\phi$. Via partially fractioning we can write every term in Eq. (A.11) as follows 
$-37-$

$$
\frac{N\left(D_{k}, s_{i}\right)}{D_{i_{1}} D_{i_{2}} D_{i_{3}} D_{i_{4}}}=\sum \frac{\tilde{N}\left(D_{k}, s_{i}\right)}{D_{j}, D_{j_{2}}}
$$

where $\mathrm{Dj}_{1}, \mathrm{Dj}_{2}$ depend on the angular variables. Examples are:

$$
\begin{aligned}
\frac{1}{N_{1} N_{2} N_{7} N_{8}}= & \frac{1}{\left(S-S_{3}\right)\left(S-S_{4}\right)}\left\{\frac{1}{N_{1} N_{2}}+\frac{1}{N_{2} N_{8}}+\frac{1}{N_{1} N_{7}}+\frac{1}{N_{7} N_{8}}\right\}(A .14) \\
\frac{1}{N_{1} N_{8} N_{3} N_{5}}= & \frac{1}{\left(S_{-}-S_{3}\right)\left(S_{3}+S_{4}-2 S^{\prime}\right)}\left\{\frac{1}{N_{1} N_{3}}+\frac{1}{N_{1} N_{5}}+\frac{1}{N_{3} N_{8}}+\frac{1}{N_{5} N_{8}}\right\}(A .15) \\
\frac{1}{N_{1} N_{2} N_{3} N_{5}}= & \frac{1}{\left(S_{3}-S^{\prime}\right)\left(S_{4}-S^{\prime}\right)} \frac{1}{N_{1} N_{2}}+\frac{1}{\left(S_{3}-S^{\prime}\right)\left(S_{3}+S_{4}-2 S^{\prime}\right)}\left\{\frac{1}{N_{1} N_{3}}\right. \\
& \left.-\frac{1}{N_{2} N_{3}}\right\}+\frac{1}{\left(S_{4}-S^{\prime}\right)\left(S_{3}+S_{4}-2 S^{\prime}\right)}\left\{\frac{1}{N_{2} N_{5}}-\frac{1}{N_{1} N_{5}}\right\}
\end{aligned}
$$

Following the above procedure the expression (A.8) can be decomposed into a series of scalar integrals which are denoted by

$$
J_{i, j}^{(n, m)}=\int d s_{3} d s_{4} N\left(s_{3}, s_{4}\right) \int d \Omega \frac{1}{\left(N_{i}\right)^{n}\left(N_{j}\right)^{m}}
$$

The angular part of the integral in Eq. (A.17) is defined by

$$
I_{i, j}^{(n, m)}=\int d \Omega \frac{1}{\left(N_{i}\right)^{n}\left(N_{j}\right)^{m}}=\int_{-1}^{1} d \cos \varphi \int_{0}^{2 \pi} d \varphi \frac{1}{(a+b \cos \vartheta)^{n}(A+B \cos \vartheta+C \sin \vartheta \cos \varphi)^{m}}
$$

where $a, b, A, B$ and $C$ are functions of $s_{i}, s$ and $s^{\prime}$. A table of the above angular integrals is given in Refs. 14) and 38). After having performed the integrals over the solid angle we can make some approximations to $J_{j l}, j_{2}$ by neglecting $\mathrm{m}^{2}$ with respect to $s_{i}$. However, care has to be taken because if powers of $\mathrm{m}^{2} / \mathrm{s}_{i}$ are prematurely neglected, one encounters spurious singularities in the integrals at $s_{i}=s$ or $s_{i}=s^{\prime}$. The most important of the integrals appearing in our calculation are 
$-39-$

$$
I_{1,3}^{(2,1)}=\frac{4 \pi}{s^{2} m^{2}} \frac{1}{x(1-x)(\rho+\alpha)}\left[-1+\frac{\gamma-\alpha}{2 \sqrt{\rho(\rho+\alpha)}} \ln \left\{\frac{\rho+\gamma+\sqrt{\rho(\rho+\alpha)}}{\rho+\gamma-\sqrt{\rho(\rho+\alpha)}}\right\}\right]_{(\mathrm{A}, 26)}
$$

$$
\begin{aligned}
& I_{1,3}^{(1,2)}=\frac{4 \pi}{s^{3}} \frac{1-x}{x^{2} \rho(\rho+\alpha)}\left[\frac{2 \rho(y-\alpha)}{\rho(2 y-\alpha)+y^{2}}-\frac{\rho}{\sqrt{\rho(\rho+\alpha)}} \ln \left\{\frac{\rho+y+\sqrt{\rho(\rho+\alpha}}{\rho+y-\sqrt{\rho / \rho+\alpha}}\right\}\right] \\
& I_{1,3}^{(2,1)}=\frac{4 \pi}{s^{3} m^{2}} \frac{1}{x \rho(\rho+\alpha)}\left[1+\frac{\gamma^{2}}{\rho(2 \gamma-\alpha)+\gamma^{2}}-\frac{3 \alpha}{\rho+\alpha}+\right. \\
& \left.\left(\frac{\gamma}{2 \sqrt{\rho(\rho+\alpha)}}-\frac{3 \rho^{2}(\gamma-\alpha)}{2[\rho(\rho+\alpha)]^{3 / 2}}\right) \ln \left\{\frac{\rho+\gamma+\sqrt{\rho(\rho+\alpha)}}{\rho+\gamma-\sqrt{\rho(\rho+\alpha}}\right]\right]
\end{aligned}
$$

Here we have introduced the following definitions.

$$
\begin{aligned}
& x=53 / 5 \quad y=54 / 3 \quad \rho=5,1, \quad \rho^{\prime}=z / x-y \\
& \alpha=\frac{4 m^{2}}{5} \frac{(1-x)(x-z)}{x^{2}} \quad y=\frac{2 m^{2}}{5} \frac{(1-x)(1-z)}{x}
\end{aligned}
$$

Insertion of the above integrals into Eq. (A.17) provides us with the final expression for the terms in $d \sigma / d s^{\prime}$. The most important full scalar ( $\phi^{3}$ type) integrals obtained from the graphs in Fig. 4 are listed below.

$$
\begin{aligned}
\int \operatorname{dps} \frac{1}{N_{1} N_{2} N_{7} N_{0}}= & \frac{16 \pi}{s^{2}(1-z)}\left[\left\{2 \ln \frac{1-z}{\varepsilon}-\frac{1}{2} \ln z\right\} \ln ^{2} \frac{s}{m^{2}}-\frac{1}{2} \ln ^{2} z \ln \frac{s}{m^{2}}-S_{3_{2}}(1-z)\right. \\
& \left.-\ln z \operatorname{li}{ }_{2}(1-z)-\frac{1}{6} \ln ^{3} z\right]
\end{aligned}
$$




$$
\begin{aligned}
& I_{1,2}^{(1,1)}=\frac{8 \pi}{s^{2} \times \sqrt{\rho^{\prime}\left(\rho^{\prime}+\alpha\right)}} \ln \left\{\frac{\rho^{\prime}+\alpha / 2+\sqrt{\rho^{\prime}\left(\rho^{\prime}+\alpha\right)}}{\alpha / 2\left(1-\frac{x \rho^{\prime}}{x-z}\right)}\right\} \\
& I_{1,7}^{(1,1)}=\frac{8 \pi}{s^{2} \sqrt{\rho\left(\rho+\alpha x^{2}\right)}} \ln \left\{\frac{\rho+\alpha x^{2} / 2+\sqrt{\rho\left(\rho+\alpha x^{2}\right)}}{\frac{\alpha x^{2}}{2}\left(1+\frac{\rho}{x-z}\right)}\right\} \\
& I_{1,3}^{(1,1)}=\frac{4 \pi}{s^{2} x \sqrt{\rho(\rho+\alpha)}} \ln \left\{\frac{\rho+\gamma+\sqrt{\rho(\rho+\alpha)}}{\rho+\gamma-\sqrt{\rho(\rho+\alpha)}\}}\right. \\
& I_{1,5}^{(1,1)}=\frac{4 \pi}{s^{2}} \frac{1}{\sqrt{\{(1-z)(1-x)-\rho\}^{2}+4 \rho \frac{m^{2}}{3}\{(1-x)(x-z-\rho x\}}} \\
& \quad \times \ln \left\{\frac{\left[(1-x)(1-z)-\rho+\frac{2 m^{2}}{3} \rho x+\sqrt{\{(1-z)(1-x)-\rho\}^{2}+4 \rho^{\frac{m}{3}}\{(1-x)(x-z-\rho x]}\right]}{\frac{4 m^{2}}{s}(1-x)^{2}\left\{\rho z+\frac{\left.m^{2}(1-z)^{2}\right\}}{3}\right\}}\right.
\end{aligned}
$$

$$
I_{1,2}^{(2,1)}=\frac{4 \pi}{5^{2} m^{2}} \frac{1}{\left(\rho^{\prime}+\alpha\right)(1-x)}\left[\frac{-1}{x-z-\beta^{\prime} x}-\frac{1}{2(x-z)} \frac{\alpha}{\sqrt{\rho^{\prime}\left(\rho^{\prime}+\alpha\right)}} \ln \left\{\frac{\rho^{\prime}+\frac{\alpha}{2}+\sqrt{\rho^{\prime}\left(\rho^{\prime}+\alpha\right)}}{\frac{\alpha}{2}\left(1-\frac{x \rho^{\prime}}{x-z}\right)}\right\}\right]
$$

$$
I_{1,2}^{(2,2)}=\frac{8 \pi}{s^{3} m^{2}} \frac{1}{x^{2} \rho^{\prime}\left(\rho^{\prime}+\alpha\right)}\left[1-\frac{3}{2} \frac{\alpha}{\rho^{\prime}+\alpha}+\left(\frac{\frac{1}{4} \alpha}{\sqrt{\rho^{\prime}\left(\rho^{\prime}+\alpha\right)}}+\frac{\frac{3}{4} \rho^{\prime 2}}{\left[\rho^{\prime}\left(\rho^{\prime}+\alpha\right)\right]^{3 / 2}}\right) \ln \left\{\frac{\rho^{\prime}+\frac{\alpha}{2}+\sqrt{\rho^{\prime}\left(\rho^{\prime}+\alpha\right)}}{\frac{\alpha}{2}\left(1-\frac{x \rho^{\prime}}{x-z}\right)}\right\}\right]
$$

$$
I_{1,7}^{(2,1)}=\frac{4 \pi}{S^{2} m^{2}} \frac{1}{\left(\rho+\alpha x^{2}\right)(1-x)}\left[\frac{-1}{\rho+x-z}-\frac{1 / 2}{x-z} \frac{\alpha x^{2}}{\sqrt{\rho\left(\rho+\alpha x^{2}\right)}} \ln \left\{\frac{\rho+\frac{1}{2} \alpha x^{2}+\sqrt{\rho\left(\rho+\alpha x^{2}\right)}}{\frac{1}{2} \alpha x^{2}\left(1+\frac{\rho}{x-z}\right)}\right\}\right]
$$


$-40-$

$$
\begin{aligned}
& \int d p s \frac{1}{N_{1} N_{3} N_{s} N_{8}}=\frac{8 \pi}{3^{2}(1-z)}\left[-\frac{2}{3} \ln ^{3} \frac{s z}{m^{2}}+2 \ln \frac{1-z}{\varepsilon} \ln ^{2} \frac{s}{m^{2}}+\left\{-h_{2}(1-z)-23(2)\right.\right. \\
& \left.\left.+\frac{3}{2} \ln ^{2} z\right\} \ln \frac{5}{m^{2}}+23(3)+\frac{2}{3} \ln ^{3} z\right] \\
& \int d p s \frac{1}{N_{1} N_{2} N_{3} N_{s}}=\frac{8 \pi}{s^{2} z(1-z)}\left[\frac{2}{3} \ln ^{3} \frac{s z}{m^{2}}-\frac{1}{2} \ln z \ln ^{2} \frac{s}{m^{2}}+\left\{h_{2}(1-z)+23(2)\right.\right. \\
& \left.\left.-\ln ^{2} z\right] \ln \frac{5}{m^{2}}-S_{12}(1-z)-23(3)-\frac{1}{2} \ln ^{3} z+23(2) \ln z\right]
\end{aligned}
$$

$$
\begin{aligned}
\int \operatorname{dps} \frac{1}{N_{1} N_{3} N_{7} N_{p}}=\frac{4 \pi}{\operatorname{sm}^{2}(1-z)}[ & -\frac{3}{2} \ln ^{2} \frac{s}{m^{2}}+\left(2 \ln \frac{1-z}{\varepsilon}-2 \ln z+2\right) \ln \frac{s}{m^{2}} \\
& \left.+3(2)-\frac{1}{1-z} \ln ^{2} z\right]
\end{aligned}
$$

$$
\begin{aligned}
\int d p s \frac{1}{N_{1}^{2} N_{2} N_{3}}=\frac{4 \pi}{s m^{2} z(1-z)} & {\left[\frac{3}{2} \ln ^{2} \frac{s}{m^{2}}+\left\{2 z \ln \frac{1-z}{\varepsilon}+(3-2 z) \ln z-2\right\} \ln \frac{s}{m^{2}}\right.} \\
& -2 \ln z+(1-2 z) 3(2)+2(1-z) \lim (1-z) \\
& \left.+(3 / 2-z) \ln ^{2} z\right]
\end{aligned}
$$

$$
\text { Sdps } \begin{aligned}
\frac{1}{N_{1} N_{3}{ }^{2} N_{7}}=\frac{8 \pi}{m^{4}} & {\left[\frac{1}{3} 3(2)+\frac{1}{3} \operatorname{lin}(1-z)+\frac{1}{6} \ln ^{2} z+\frac{1}{3(1-z)} \ln z\right.} \\
& \left.-\frac{1}{6} \frac{1}{1-z}\left\{1+\frac{2}{1-z} \ln z+\frac{1}{(1-z)^{2}} \ln ^{2} z\right\}+\frac{1}{2} \frac{1}{1-z}\right]
\end{aligned}
$$

(A.36) 
$-41-$

$$
\int \operatorname{dps} \frac{1}{N_{1}{ }^{2} N_{2}{ }^{2}}=\frac{4 \pi}{m^{4}(1-z)}\left[2 \ln \frac{1-z}{\varepsilon}-\ln z\right]
$$

$$
\int \operatorname{dps} \frac{1}{N_{1}{ }^{2} N_{3}{ }^{2}}=\frac{4 \pi}{m^{4}(1-z)}\left[\ln \frac{1-z}{\varepsilon}-\ln z-1\right]
$$

where $d p s=d s_{3} d s_{4} d \Omega$. 


\section{APPENDIX B}

In the previous Appendix, we have discussed the hard integrals. Their calculation, although tedious, is straightforward. The soft-photon integrals, however, are easier to compute, although they are more tricky, as we will see below.

In the case that one photon is soft and the other one is hard, we have the following kinematical boundaries for the integral in Eq. (A.8).

I photon 1 is soft $\omega_{1}\left\langle\Delta\right.$ and photon 2 is hard $\left.\omega_{2}\right\rangle \Delta$

$$
s(1-\varepsilon)<s_{3}<(\sqrt{s}-\lambda)^{2} \quad s \frac{s^{\prime}}{s_{3}}<s_{4}<s+s^{\prime}-s_{3}
$$

II photon 2 is soft $\omega_{2}\left\langle\Delta\right.$ and photon 1 is hard $\omega_{1}>\Delta$

$$
s(1-\varepsilon)<s_{4}<(\sqrt{5}-\lambda)^{2} \quad s \frac{s^{\prime}}{s_{4}}<s_{3}<s+s^{\prime}-s_{4}
$$

where $\varepsilon=2 \Delta / \sqrt{ }$ s as defined before and $\lambda$ is the photon regulator mass which we have to introduce in order to regulate the infra-red divergences ${ }^{*}$. If we choose $\Delta \ll m$ we can apply the soft-photon approximation to the matrix element $T^{(2)}$, Eq. (A.3). It appears that $\mid \mathrm{T}^{(2)} 1^{2}$ will factorize as follows

I $\quad k_{1} \rightarrow 0 \quad\left|T^{(2)}\left(k_{1}, k_{2}\right)\right|^{2}=, S\left(k_{1}\right)\left|T^{(1)}\left(k_{2}\right)\right|^{2}$

II $k_{2} \rightarrow 0 \quad\left|T^{(2)}\left(k_{1}, k_{2}\right)\right|^{2}=S\left(k_{2}\right)\left|T^{(1)}\left(k_{1}\right)\right|^{2}$

Here $s\left(k_{i}\right)$ is given by the well-known soft-photon approximation of the amplitude where one photon is emitted in the final state ${ }^{16)}$

*) Notice that in the case of $\lambda \neq 0, \quad \omega_{1}=\frac{s-s_{3}+\lambda^{2}}{2 \sqrt{s}}, \omega_{2}=\frac{s-s_{4}+\lambda^{2}}{2 \sqrt{s}}$ 


$$
S\left(k_{0}\right)=\left(\frac{p_{+\mu}}{p_{+} \cdot k_{1}}-\frac{p_{-\mu}}{p_{-} \cdot k_{1}}\right)^{2}
$$

By inserting of Eq. (B.3) into Eq. (A.18) and using the boundary conditions above we obtain

$$
\frac{d \sigma}{d s^{\prime}}=\sigma^{(0)}\left(s^{\prime}\right) \frac{e^{4}}{64(2 n)^{5} s} \int_{s(1-\varepsilon)}^{(\sqrt{s}-\lambda)^{2}} d s_{3} d \Omega_{1} S\left(k_{1}\right) \int_{s^{\prime} / s_{3}}^{s+s^{\prime}-s_{3}} d s_{4} d \Omega_{2}\left|T^{(1)}\left(k_{2}\right)\right|^{2}
$$

Notice that the hard and the soft photons are uncorrelated in phase space as long as $\omega_{1}<\Delta$. This holds for the solid angles $d \Omega_{i}$ as well as the energies $s_{i}$. The latter can be explicitly shown by partially integrating expression (B.6) with respect to $s_{3}$. The same holds if the rôles of $k_{1}$ and $k_{2}$ are interchanged. Addition of both contributions yields the expression in Eq. (2.25). Another way to calculate expression (2.25) which reveals the factorization of the phase space more clearly is the following. Introduce photon regulators in the on-mass shell $\delta$-functions of Eq. (A.2) and replace $T^{(2)}$ by Eq. (B.3). Then in the 1 imit $k_{1} \rightarrow 0$ we get

$\frac{d \sigma}{d s^{\prime}}=\sigma^{\prime \prime \prime}\left(s^{\prime}\right) \frac{e^{4}}{8(2 \pi)^{b_{s}} \int_{0}^{\Delta}} \frac{d^{3} k_{1}}{2 \sqrt{\left(k_{1}\right)^{2}+\lambda^{2}}} S\left(k_{1}\right) \int d^{4} k_{2} d^{4} q^{+} \delta^{+}\left(q^{2}-s^{\prime}\right) \delta^{+}\left(k_{2}^{2}\right) \delta^{4}\left(p_{1}+p_{2}-q^{-}-k_{2}\right)\left|T^{(1)}\left(k_{2}\right)\right|^{2}$

One recognizes immediately that the first factor is just given by

$$
\frac{e^{2}}{(2 \pi)^{3}} \int_{0}^{\Delta} \frac{d^{3} k_{1}}{2 \sqrt{\left|k_{1}\right|^{2}+\lambda^{2}}} S\left(k_{1}\right)=-\delta_{1}^{S}(\lambda, \varepsilon)
$$

which is the well-known bremstrablung factor given in Eq. (2.12). In the case that two soft photons appear in the final state, $T^{(2)}$ factorizes in the soft-photon 1 imit and we can write

$$
\left|T^{(2)}\left(k_{1}, k_{2}\right)\right|^{2}=S\left(k_{1}\right) S\left(k_{2}\right)\left|T^{(01}\right|^{2}
$$

The soft two-photon contribution to the cross-section is given by the expression [see eq. (2.36) where $m \rightarrow \lambda$ ] 


$$
\int_{s(1-\varepsilon)}^{(\sqrt{s}-2 \lambda)^{2}} d s^{\prime} \frac{d \sigma}{d s^{\prime}}
$$

Substituting Eq. (B.9) in Eq. (A. 8) and performing the integration over $s$ ' gives the expression in Eq. $(2.20)$. However, this is a very difficult procedure since the integration variables $\mathrm{s}_{3}, \mathrm{~s}_{4}$ depend in a very complicated way on the photon mass $\lambda$. Therefore we choose the second procedure as has been outlined in Eq. (B.7). In the limit $\mathrm{k}_{1}, \mathrm{k}_{2} \rightarrow 0$ and substitution of Eq. (B.9) in Eq. (A.8), the soft-photon cross-section becomes

$\frac{d \sigma}{d s^{\prime}}=\delta\left(s^{\prime}-s\right) \sigma^{(\alpha)}\left(s^{\prime}\right) \frac{e^{4}}{8(2 \pi)^{b} s} \int_{0}^{\Delta} \frac{d^{3} \vec{k}_{1}}{2 \sqrt{\left|k_{1}\right|^{2}+\lambda^{2}}} \int_{0}^{\Delta-\left|k_{1}\right| d^{3} \vec{k}_{2}} \frac{d^{2}}{2 \sqrt{\left|k_{2}\right|^{2}+\lambda^{2}}} S\left(k_{1}\right) S\left(k_{2}\right)\left|T^{(s)}\right|^{2}$

Notice that in the above expression the angular variables which correspond to the photons $k_{1}$ and $k_{2}$ are uncorrelated. However, because of the boundary condition on $s^{\prime}$ in Eq. (B.10) this does not hold for the energies $\omega_{1}$ and $\omega_{2}$ which are correlated. From $s^{\prime}>s(1-\varepsilon)$ it follows that $\omega_{1}+\omega_{2}<\Delta$. We can now rewrite the soft-photon integral in Eq. (B.11) as follows

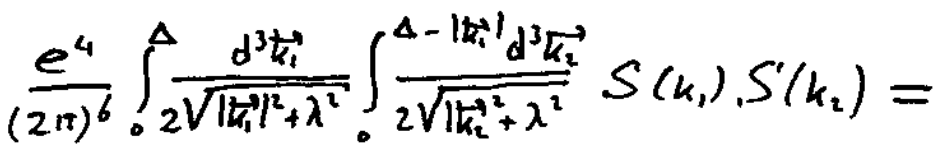

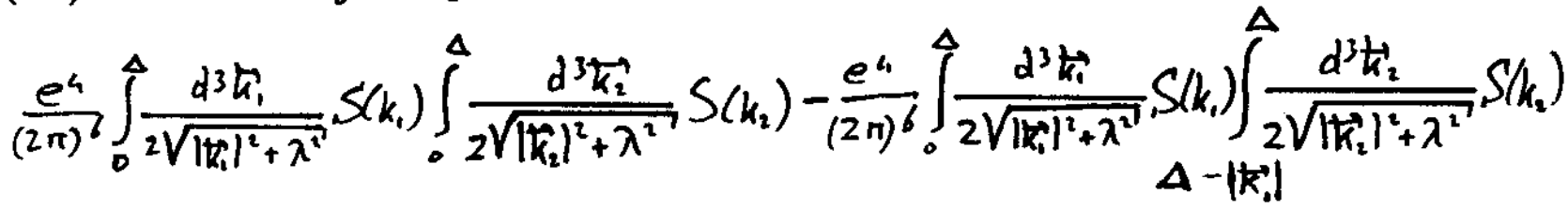

The last integral in Eq. (B.12) is infra-red finite so that we can omit $\lambda$. A straight forward calculation reveals that $\left(\left|\vec{k}_{i}\right|=\omega_{i}\right)$

$$
4\left(\frac{\alpha}{\pi}\right)^{2}\left(\ln \frac{s}{m^{2}}-1\right)^{2} \int_{0}^{\Delta} \frac{d \omega_{1}}{\omega_{1}} \int_{\Delta-\omega_{1}}^{\Delta} \frac{d \omega_{2}}{\omega_{2}}=-2 C_{2}
$$

with $\mathrm{C}_{2}$ defined in Eq. (2.21). The first part of expression (B.12) equals $\left[\delta_{1}^{S_{1}}(\lambda, \varepsilon)\right]^{2}$ so that we finally get expression $(2.20)$. 
Finally we want to comment on the value of $\Delta$, which in our calculations has been used as a cut-off on the soft and hard-photon bremstrahlung integrals. In lowest order in $\alpha$ one can show that the bremstrahlung integrals have the same form irrespective of the value of $\Delta$, i.e., $\Delta\left\langle\right.$ m or $\Delta \gg \mathrm{m}$ provided $\Delta\left\langle\mathrm{s}^{\prime} \mathrm{s}^{\prime}\right.$. If we call $\Delta=\Delta_{s}$ for $\Delta\left\langle\left\langle\mathrm{m}\right.\right.$ and $\Delta=\Delta_{\ell}$ for $\Delta \gg \mathrm{m}$, it appears ${ }^{39)}$ that the order $\alpha$ bremsstrahlung integral is equal to

$$
\frac{e^{2}}{(2 \pi)^{3}} \int_{\Delta_{s}}^{\Delta_{l}} \frac{d^{3} k}{2|\vec{k}|} S(k)=-\frac{\alpha}{\pi}\left[2 \ln \frac{\Delta_{l}}{\Delta_{s}}(L-1)\right]
$$

Addition of expression (B.14) to $-\delta_{1}^{S} l\left(\lambda, \Delta_{S}\right)$ [Eq. (B.8)] yields $-\delta_{1}^{S}{ }^{1}\left(\lambda, \Delta_{\ell}\right)$ so that $\Delta_{s}$ is effectively replaced by $\Delta_{\ell}$. This property does not hold for each order $\alpha^{2}$ bremsstrahlung integral. However, we have explicitly checked that if all integrals are added, one observes the same phenomenon. Therefore the form of $\delta_{2}^{S 2}(\lambda, \Delta)[\mathrm{Eq} \cdot(2.20)$ ] is left unchanged and is independent of the value of $\Delta$ with respect to the electron mass. 


\section{REFERENCES}

1) "Physics at LEP", eds. J. Ellis and R. Peccei - CERN Report 86-02, Vols. 1 and 2 .

2) M. Greco, G. Pancheri-Srivastava and Y. Srivastava - Nucl.Phys. B171 (1980) 118 .

3) See Ref. 1), G. A1tarel1i - LEP Report Vol. 1, 3; See Ref. 1), M. Greco - LEP Report Vo1. 1, 182.

4) G. Passarino and M. Veltman - Nuc1.Phys. B160 (1979) 151;

W. Wetze1 - Nuc1.Phys. B227 (1983) 1;

J.P. Cole - in "Radiative Corrections in SU(2) $\times U(1) "$, eds. B.W. Lynn and J.F. Wheater (World Scientific, 1984), 139;

R.W. Brown, R. Beckex and E.A. Paschos - Phys.Rev.Lett. 52 (1984) 1192 ;

M. Böhm and W. Hollik - Phys.Lett. 139B (1984) 213;

B.W. Lynn and R.G. Stuart - Nucl.Phys. B253 (1985) 216;

M. Igarashi, N. Nakazawa, T. Shimida and Y. Shimizu - Nuc1.Phys. B263 (1986) 347 .

5) F.A. Berends and R. Kleiss - Nuc1.Phys. B177 (1981) 237.

6) F.A. Berends, R. Kleiss and S. Jadach - Nucl.Phys. B202 (1982) 63.

7) See Ref. 1), R. Kleiss - Vol. 1, 153.

8) C. Maña, M. Maxtinez and F. Cornet - DESY 86-114.

9) Y.S. Tsai - SLAC-PUB-3129.

10) E.A. Kuraev and V.S. Fadin - Sov.J.Nuc1.Phys. 41 (1985) 466.

11) See Ref. 1), G. Altarelli and G. Martinelli - Vol. 1, 47.

12) 0. Nicrosini and L. Trentadue - UPRF-86-132.

13) "Contribution to the LEP 200 ECFA Workshop", A. Barroso et al. CERN-EP/87-70 (1987).

14) A.N. Schellekens and W.L. van Neerven - Phys.Rev. D21 (1980) 2619;

A.N. Schellekens - Ph.D. Thesis, University of Nijmegen (1981).

15) S. Jadach - MPI-PAE/PTh-6/87 (1987).

16) D.R. Yennie, S.C. Frautschi and H. Suura - Ann.Phys. 13 (1961) 379.

17) R. Barbieri, J.A. Mignaco and E. Remiddi - Nuovo Cimento 11A (1972) 824, 865 .

18) G.J.H. Burgers - Phys.Lett. B164 (1985) 167.

19) F.A. Berends, G.J.H. Burgers and W.L. van Neerven - Phys.Lett. Bl 77 (1986) 191 . 
20) A. Devoto and D.W. Duke - Riv. Nuovo Cimento 7, $\mathrm{N}^{\circ} 6$ (1984).

21) S. Eidelman and E. Kuraev - Nucl.Phys. B143 (1978) 353;

F.A. Berends and R. Kleiss - Nucl.Phys. B186 (1981) 22.

22) T. Kinoshita - J.Math.Phys. 3 (1962) 650;

T.D. Lee and M. Nauenberg - Phys.Rev. BI33 (1964) 1549.

23) F.A. Berends, G.J.H. Burgers and W.L. van Neerven - Phys.Lett. Bl85 (1987) 395.

24) M. Igarashi and N. Nakazawa - Nuc1.Phys. B288 (1987) 301.

25) D.R. Yennie - Phys.Rev.Lett. 34 (1975) 239;

M. Greco, G. Pancheri-Srivastava and Y. Srivastava - Phys.Lett. 56B (1975) 367;

J.D. Jackson and D.L. Scharre - Nuc1.Instrum.Methods 128 (1975) 13.

26) F. Bloch and A. Nordsieck - Phys.Rev. 52 (1977) 54.

27) G. Altarelli and G. Parisi - Nuc1.Phys. B126 (1977) 298.

28) V. Gribov and L. Lipatov - Yad.Fiz. 15 (1972) 781, 1218; Sov.J.Nucl.Phys. 15 (1972) 675, 938 .

29) H. Georgi - Phys.Rev. D17 (1978) 3010.

30) H.D. Politzer - Physics Reports 14C (1974) 129.

31) C.T.H. Davies and W.J. Stirling - Nuc1.Phys. B244 (1984) 337.

32) A.M. Mueller - Phys.Rev. D18 (1978) 3705.

33) E.G. Floratos, R. Lacaze and C. Kounnas - Phys.Lett. B98 (1981) 285.

34) E.A. Kuraev and L.N. Lipatov - Yad.Fiz. 16 (1972) 1060; Sov.J.Nuc1.Phys. 16 (1973) 584.

35) P.H.W.M. Daverveldt - Ph.D. Thesis, University of Leiden (1985); F.A. Berends, P.H.W.M. Daverveldt and R. Kleiss - Nucl.Phys. B253 (1985) 441; Comp.Phys.Comm. 40 (1986) 271 .

36) V.N. Baier, V.S. Fadin, V.A. Khoze and E.A. Kuraev - Physics Reports 78 (1980) 293.

37) A.C. Hearn - REDUCE 2, Users Manue1, University of Utah (1973).

38) A. Devoto, D.W. Duke, J.D. Kimel and G.A. Sowe11 - Phys.Rev. D30 (1984) 541 .

39) B. Humpert and W.L. van Neerven - Nucl.Phys. B184 (1981) 225. 


\section{FIGURE CAPTIONS}

Fig. I Born graph of the process $e^{+} e^{-} \rightarrow z\left(\gamma^{*}\right) \rightarrow \mu^{+} \mu^{-}$.

Fig. $20(\alpha)$ bremstrahlung graphs of the process $e^{+} e^{-} \rightarrow \gamma+z\left(\gamma^{*}\right)$.

Fig. 3 Photonic $0(\alpha)$ correction to the Born graph in Fig. 1 .

Fig. $40\left(\alpha^{2}\right)$ bremsstrahlung graphs of the process $e^{+} e^{-} \rightarrow \gamma+\gamma+z\left(\gamma^{*}\right)$.

Fig. 5 Photonic $0\left(\alpha^{2}\right)$ correction to the Born graph in Fig. 1.

Fig. $60(\alpha)$ corrections to the process in Fig. 2.

Fig. 7 Dalitz plot of the process in Fig. 4 [see Eq. (A.4)].

Fig. 8 Fermion pair production $e^{+} e^{-} \rightarrow f^{+} f^{-}+z\left(\gamma^{*}\right)$

Fig. 9 Electron-positron pair production $e^{+} e^{-} \rightarrow e^{+} e^{-}+z\left(\gamma^{*}\right)$.

Fig. 10 Fermionic $0\left(\alpha^{2}\right)$ correction to the Born graph in Fig. 1 .

Fig. $110(\alpha)$ operator matrix element corresponding to the processes in Figs. 2 and 3 .

Fig. $120\left(\alpha^{2}\right)$ operator matrix element corresponding to the processes in Figs. 4,5 and 6 .

Fig. $130\left(\alpha^{2}\right)$ operator matrix element corresponding to the process in Figs. 8 and 10 .

Fig. $140(\alpha)$ operator matrix element $\Gamma_{\text {o,er }}$ corresponding to the process in Fig. 9.

Fig. $150(\alpha)$ operator matrix element $\Gamma_{o, \gamma e}$ corresponding to the process in Fig. 9. 
Fig. $160(\alpha)$ reduced cross-section $\tilde{\sigma}_{o, e \gamma}\left(e \gamma \rightarrow e+z\left(\gamma^{*}\right)\right)$ corresponding to the process in Fig. 9.

Fig. $170\left(\alpha^{2}\right)$ operator matrix element corresponding to the process in Fig. 9.

Fig. 18 Vertex cut graph [Ref. 32)] corresponding to the interference terms of the reactions in Figs. 8 and 9 .

Fig. $19 \mathrm{~d} \sigma / \mathrm{ds}^{\prime}$ at $\sqrt{s}=100 \mathrm{GeV}$ for the photonic process $I$ and the non-photonic processes II (electrons on $1 y$ ) and III+IV. $M_{Z}=93 \mathrm{GeV}, \Gamma_{Z}=2.5 \mathrm{GeV}$, $\sin ^{2} \theta_{W}=0.223$. Al so plotted is $d \sigma / d s^{\prime}$ for multiperipheral muon-pair production (dashed line).

Fig. 20 Multiperipheral graphs for the two-photon process $e^{+} e^{-} \rightarrow e^{+} e^{-} \mu^{+} \mu^{-}$.

Fig. $21 W\left(s^{\prime}\right)=\left(s / \sigma_{0}\left(s^{\prime}\right)\right) \mathrm{d} \sigma / \mathrm{ds} s^{\prime}$ at $\checkmark s=100 \mathrm{GeV}$. Dashed Iine: $0(\alpha)$, solid line: $O\left(\alpha^{2}\right)$ corrected.

Fig. 22 Total cross-section for muon-pair production for $M_{z}=93$ GeV, $\Gamma_{Z}=2.5 \mathrm{GeV}, \sin ^{2} \theta_{\mathrm{W}}=0.223$. Dotted line: lowest order, dashed Iine: $O(\alpha)$ corrected, solid 1 ine: $O\left(\alpha^{2}\right)$ corrected.

Fig. 23 The influence of initial-state radiation on the shape of the Z-peak. $M_{Z}=93 \mathrm{GeV}, \Gamma_{Z}=2.5 \mathrm{GeV}, \sin ^{2} \theta_{W}=0.223$. Dashed line: $O(\alpha)$ corrected, dotted line: $O(\alpha)$ corrected + soft-photon resummation, dash-dotted line: $0\left(\alpha^{2}\right)$ corrected, solid line: $O\left(\alpha^{2}\right)$ corrected + soft-photon resummation.

Fig. 24 Soft-photon approximation of the $z$-peak. $M_{z}=93 \mathrm{GeV}, \Gamma_{z}=2.5 \mathrm{GeV}$, $\sin ^{2} \theta_{W}=0.223$. Upper-dashed Iine: Gribov-Lipatov 28$)$, Eq. (3.10), lower-dashed Iine, Eq. (3.13). For comparison, the $O\left(\alpha^{2}\right)$ corrected + soft-photon resummation curve from Fig. 23 has also been plotted. 


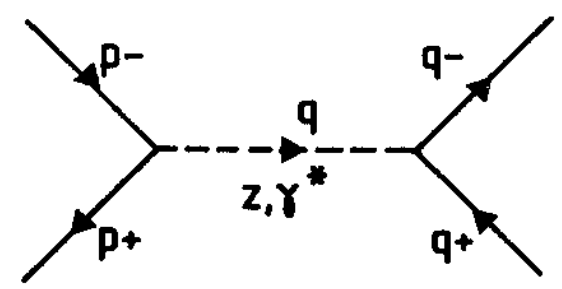

Fig. 1
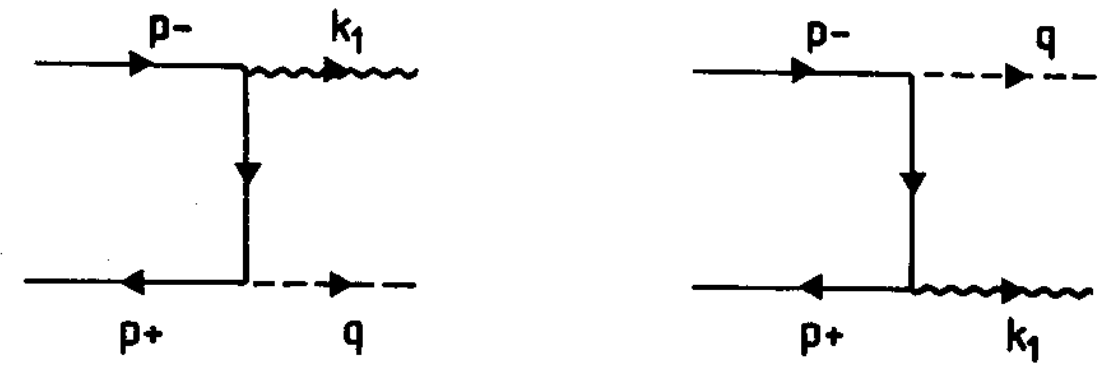

Fig. 2
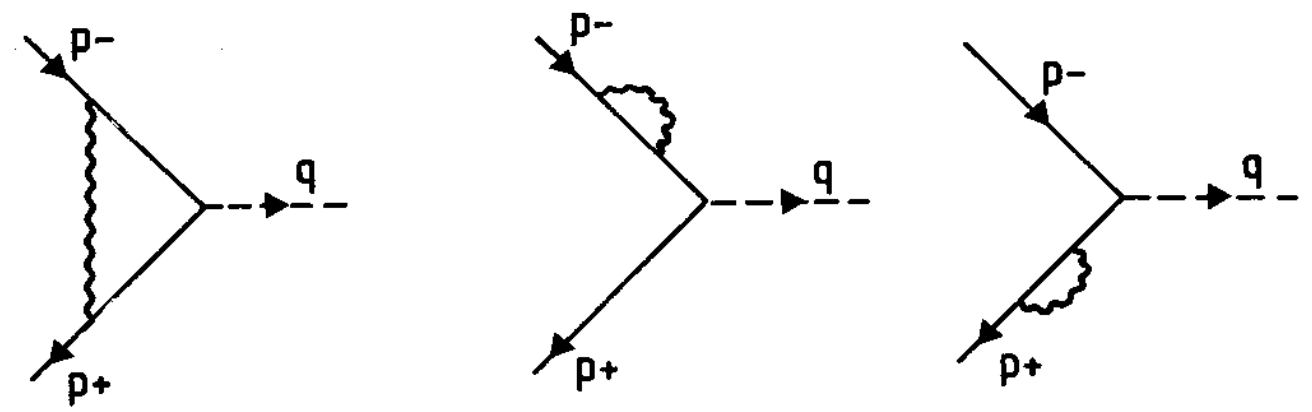

Fig. 3 

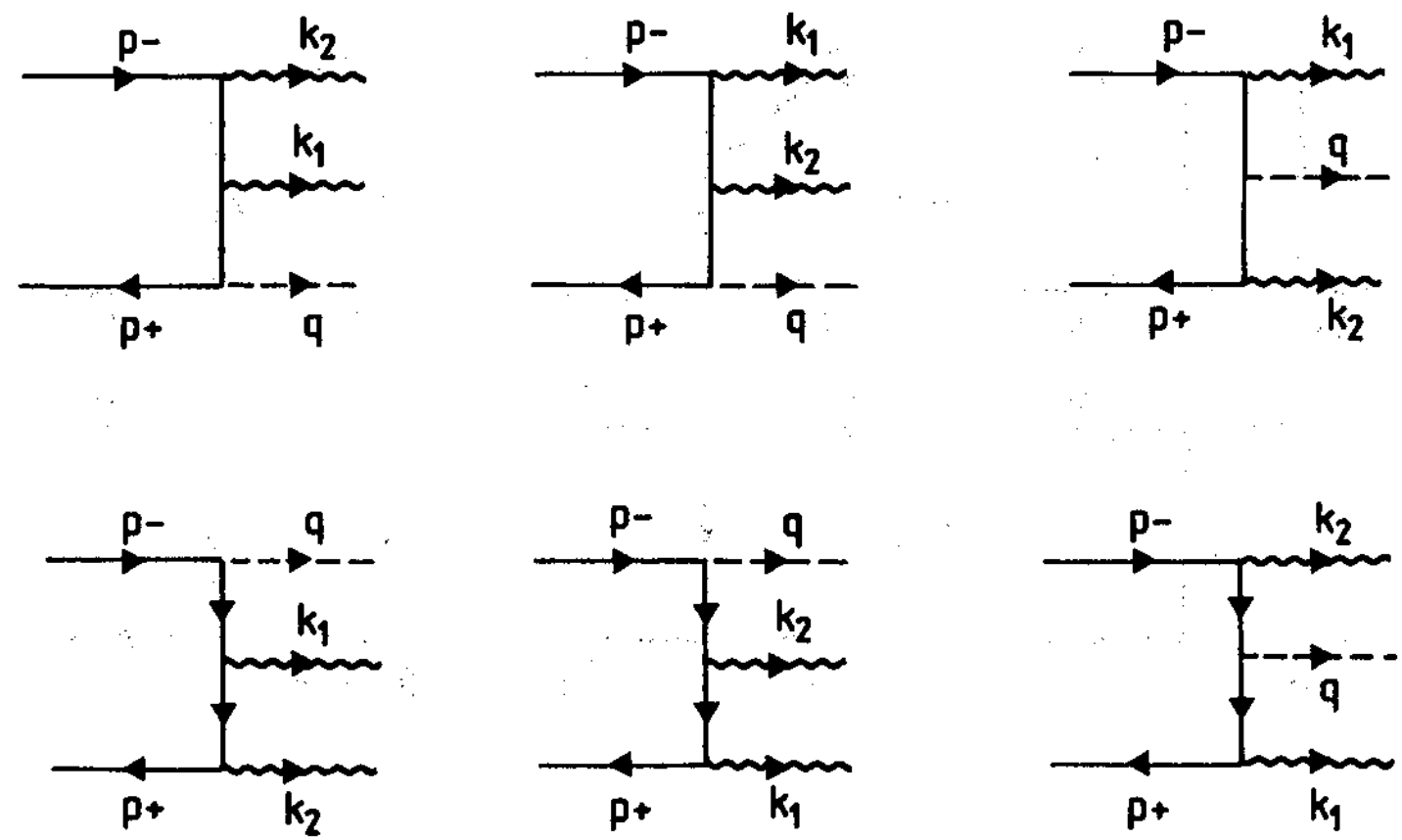

Fig. 4
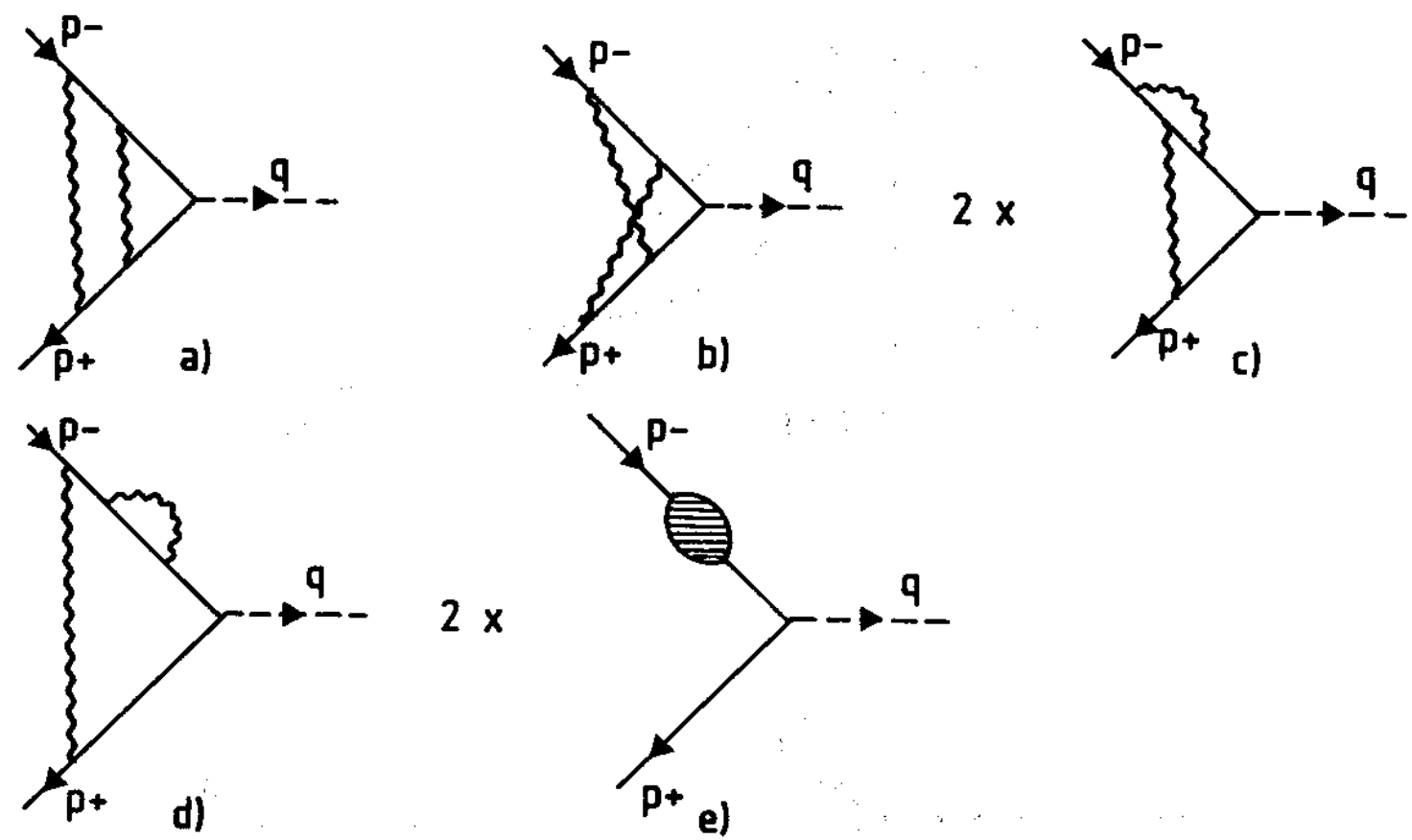

Fig. 5 

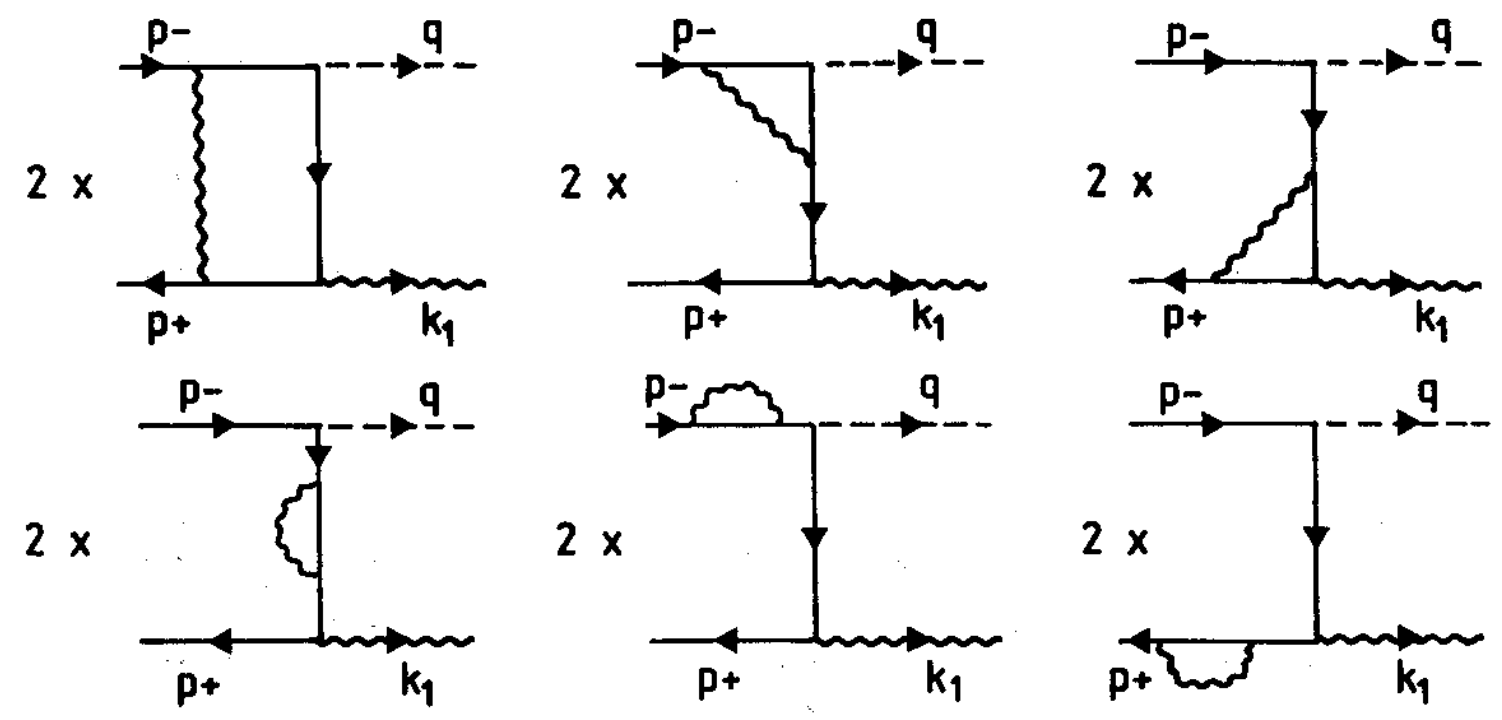

Fig. 6

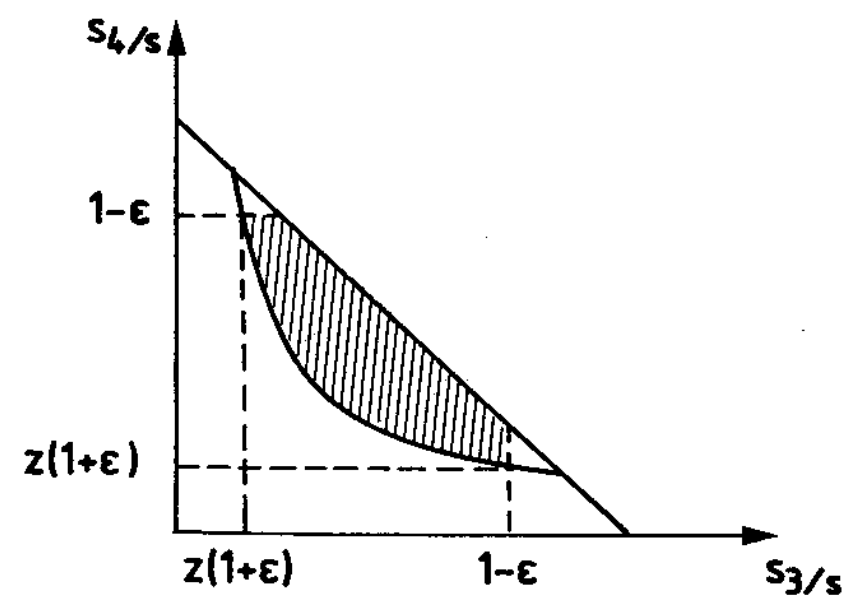

Fig. 7
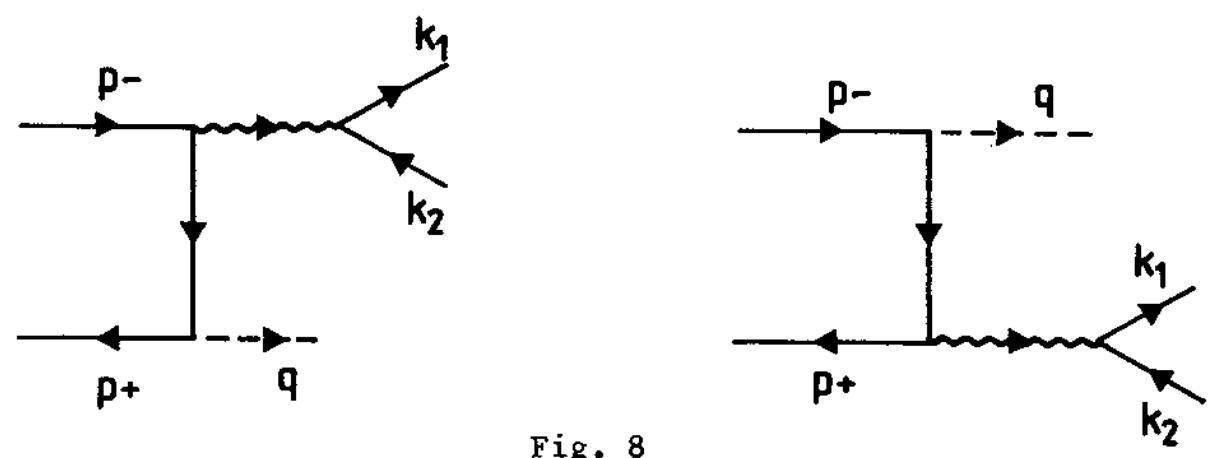

Fig. 8 

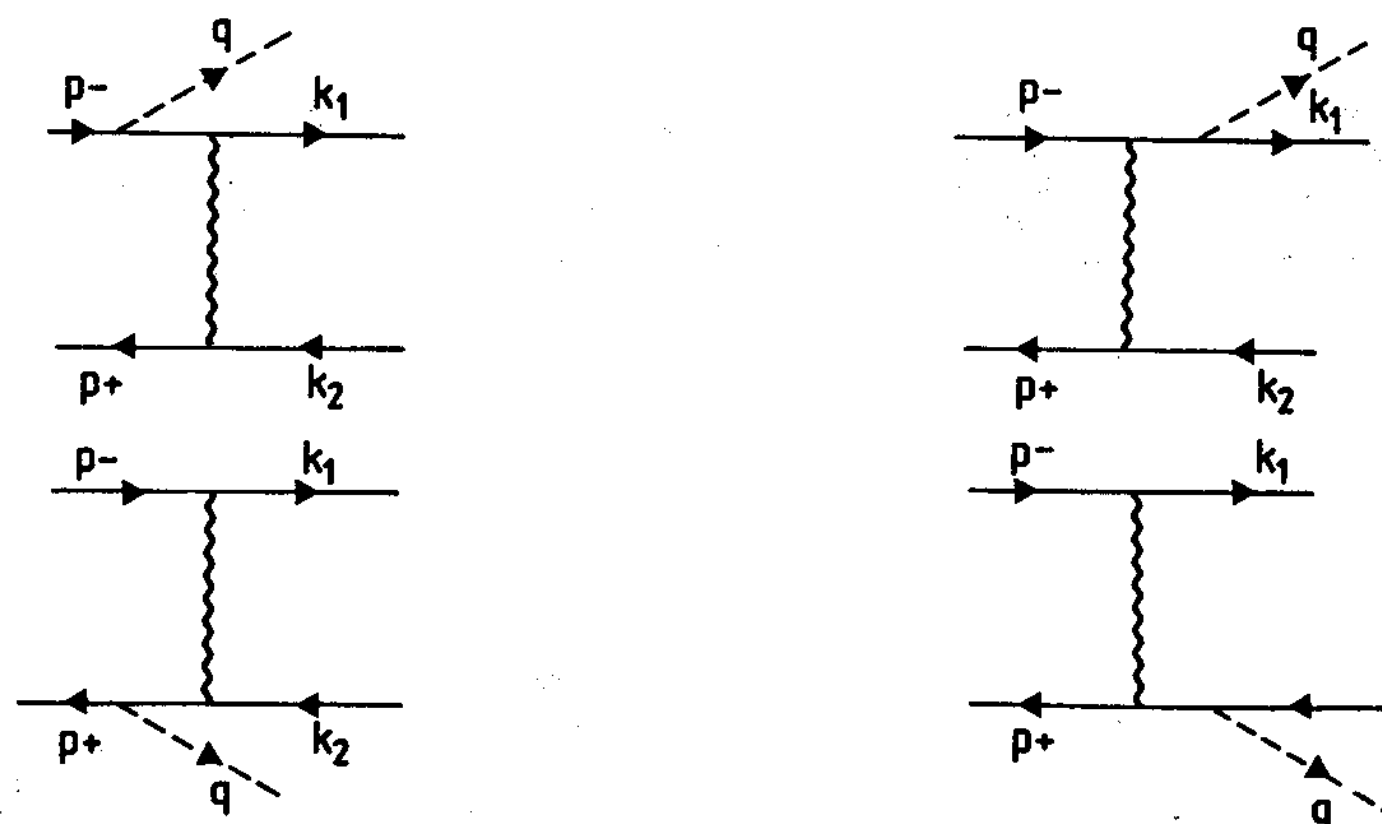

Fig. 9
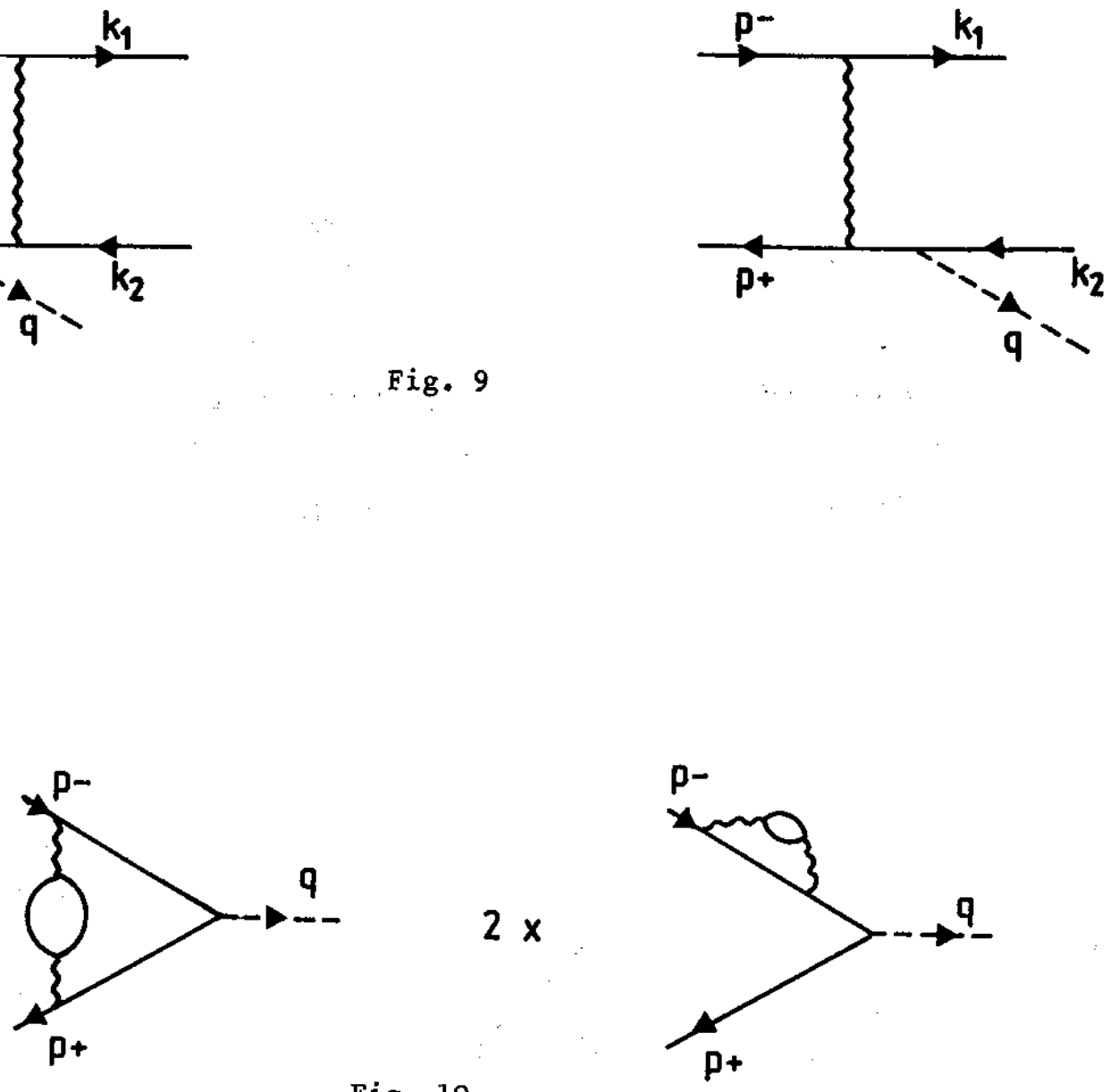

Fig. 10
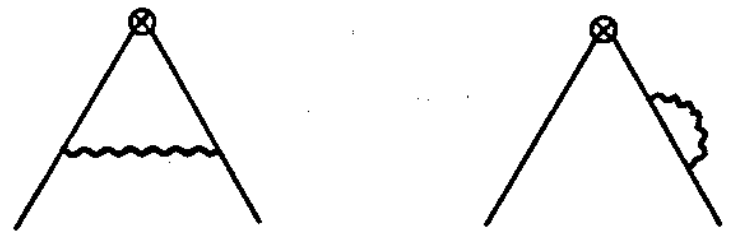

Fig. 11 

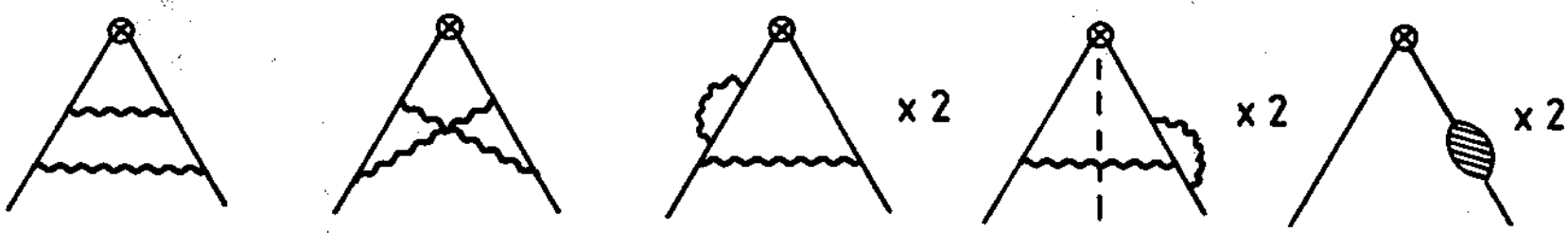

Fig. 12

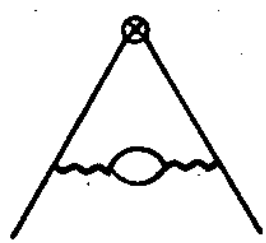

Fig. 13

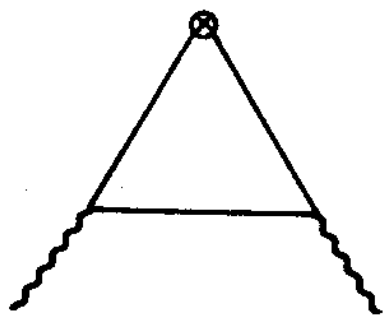

Fig. 14

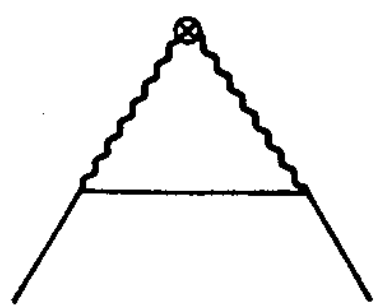

Fig - 15

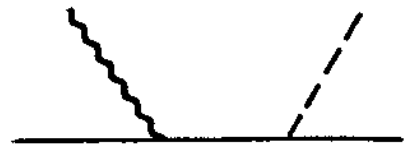

Fig. 16
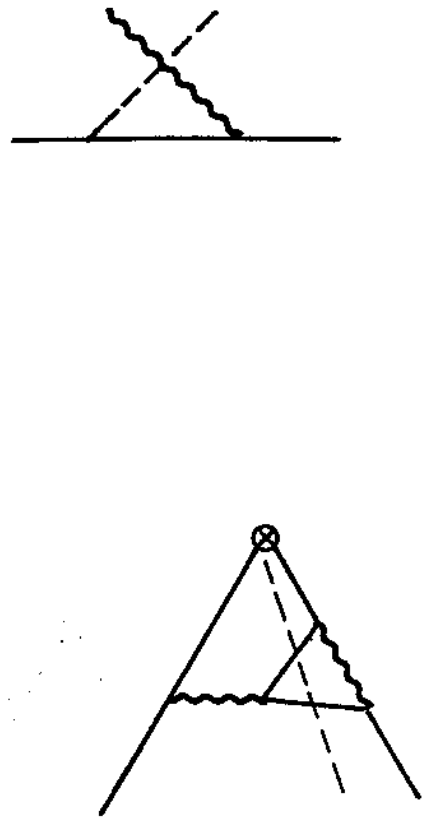

Fig. 18

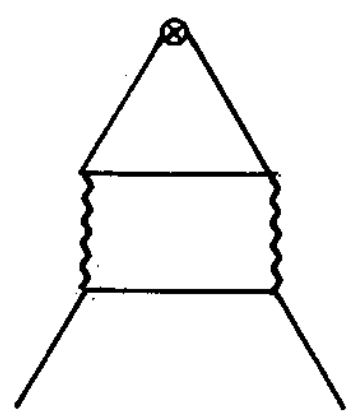

Fig. 17 


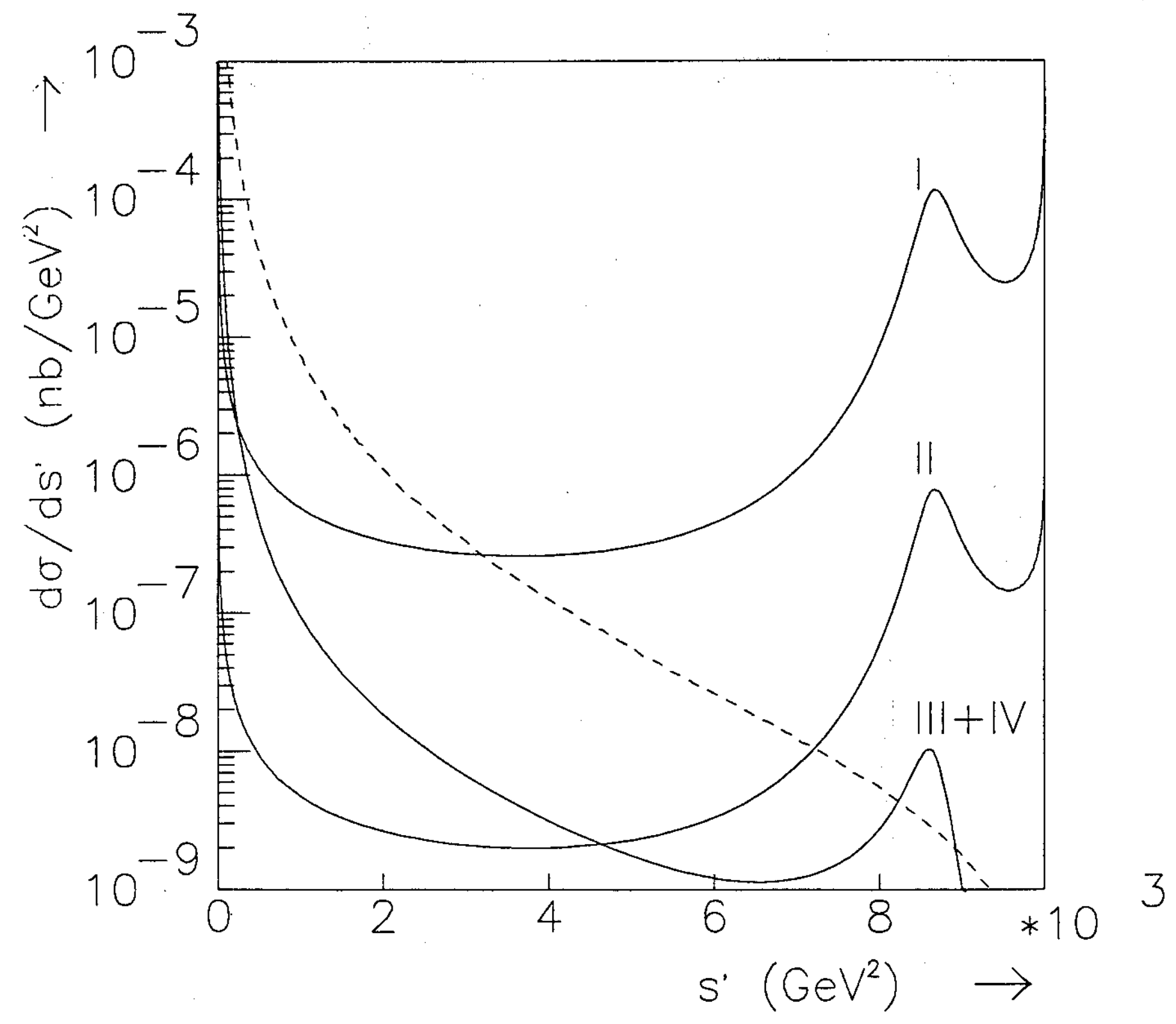

Fig. 19 

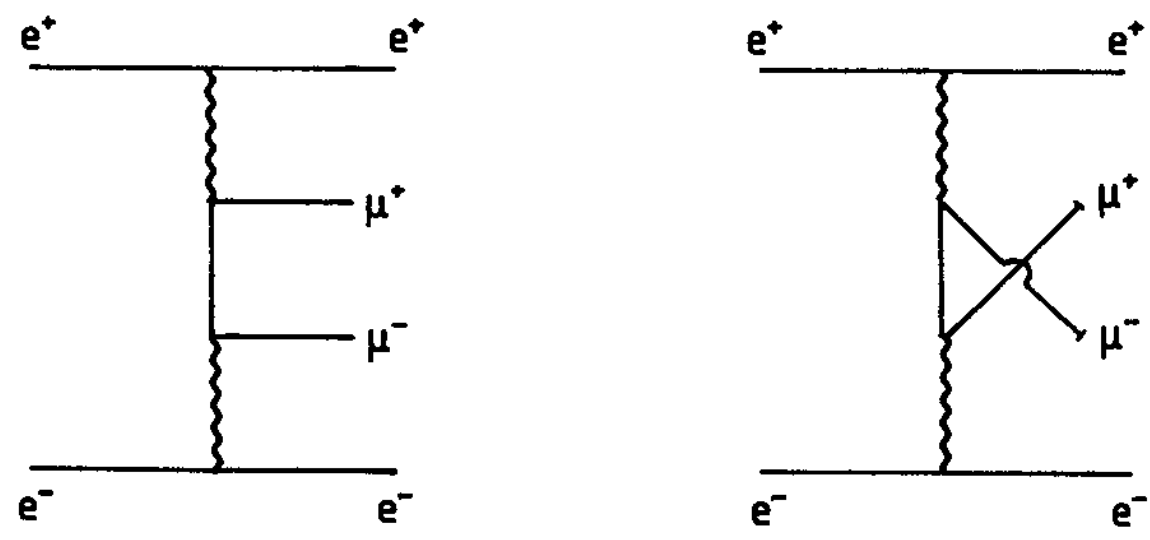

Fig. 20

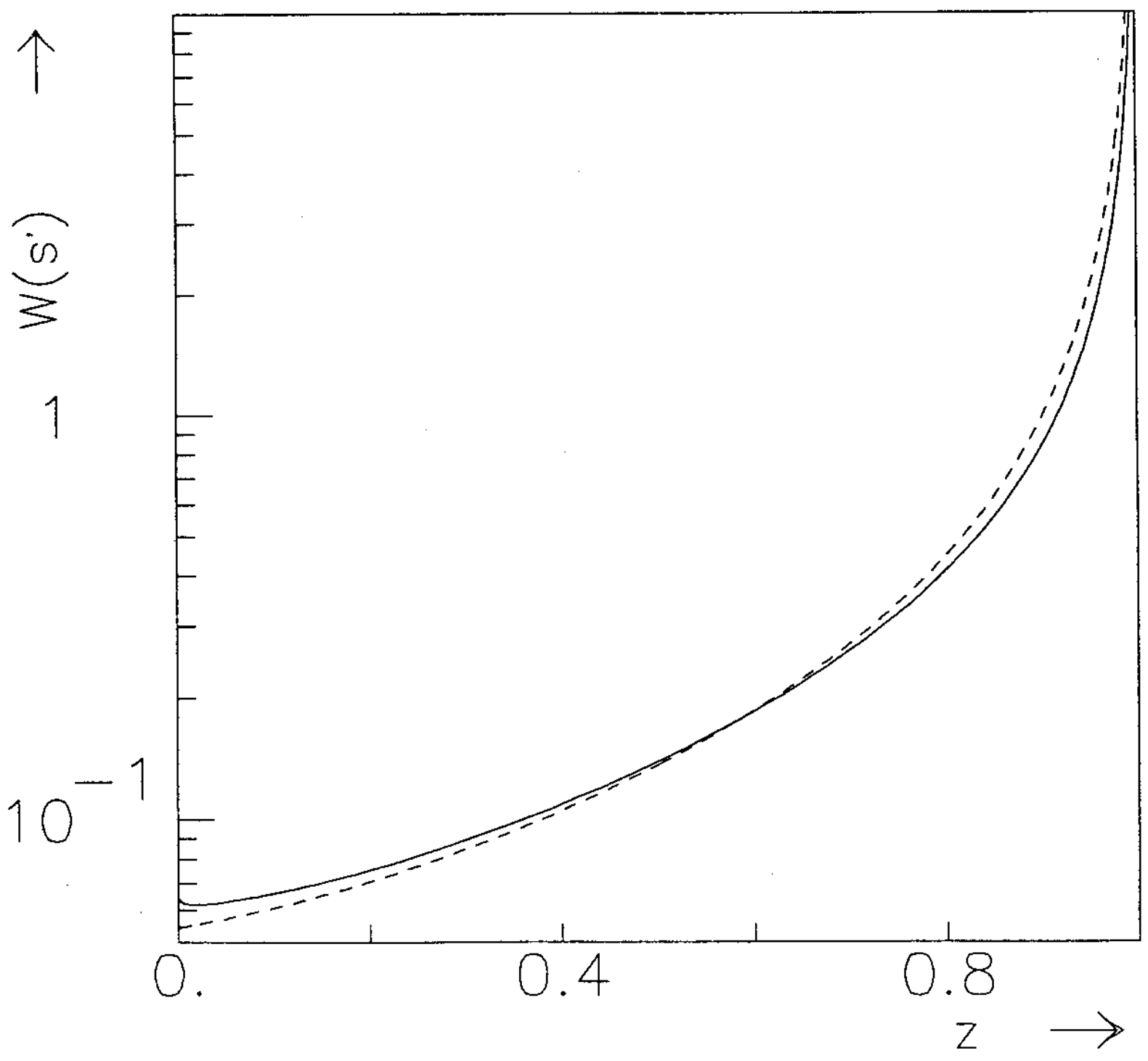

Fig. 21 


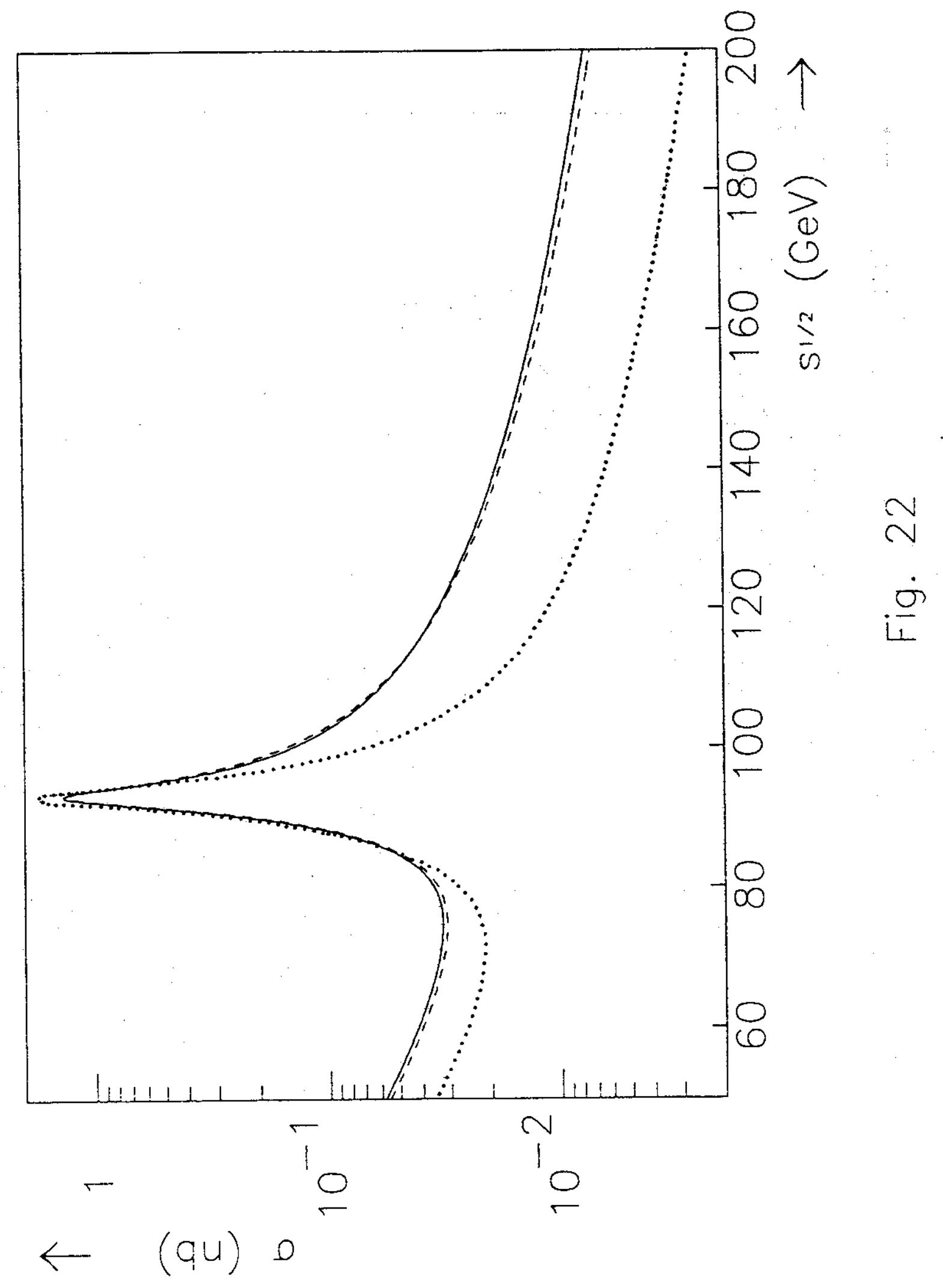




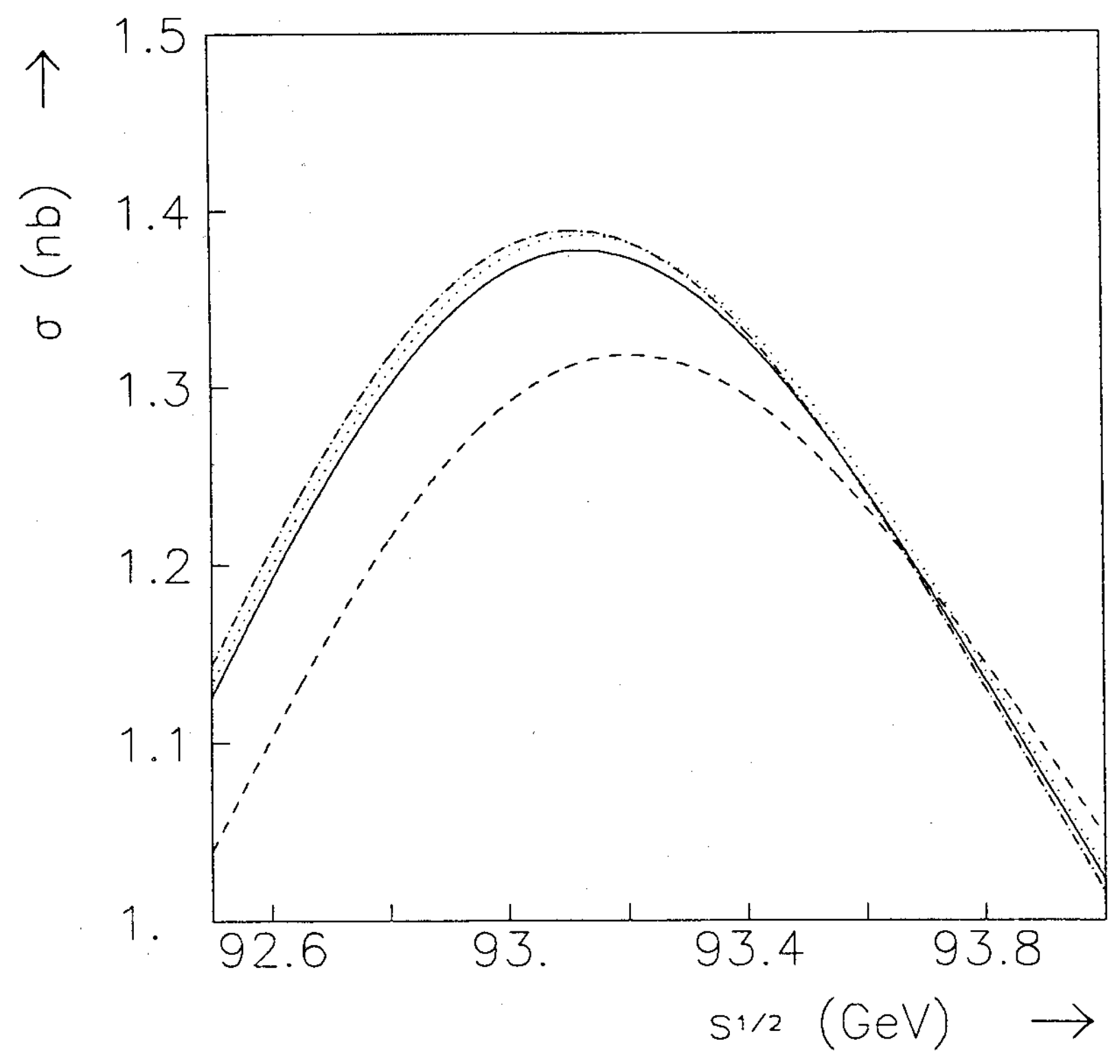

Fig. 23 


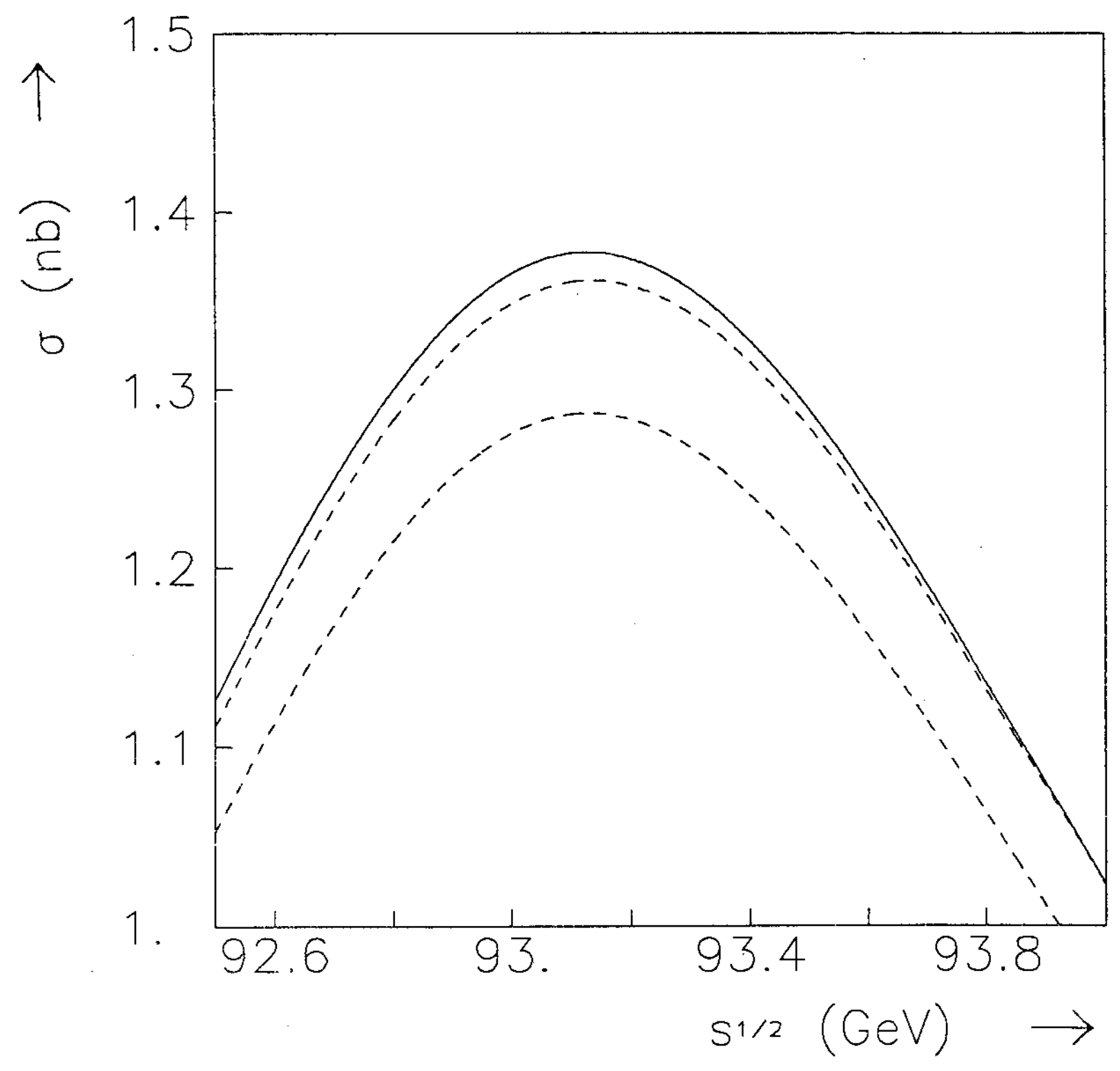

Fig. 24 